\title{
The active space debris removal mission RemoveDebris. Part 1: from concept to launch
}

\author{
Jason L. Forshaw', Guglielmo S. Aglietti², Simon Fellowes \\ Surrey Space Centre, University of Surrey, Guildford, UK \\ Thierry Salmon \\ Ariane Group, Bordeaux, France
}

Ingo Retat ${ }^{a}$, Alexander Hall ${ }^{b}$, Thomas Chabot ${ }^{c}$, Aurélien Pisseloup ${ }^{c}$

Airbus: ${ }^{a}$ Bremen, Germany; ${ }^{b}$ Stevenage, UK; ${ }^{c}$ Toulouse, France

Daniel Tye

Surrey Satellite Technology Limited (SSTL), Guildford, UK

Cesar Bernal $^{f}$, François Chaumette $^{g}$, Alexandre Pollini ${ }^{h}$, Willem H. Steyn ${ }^{i}$

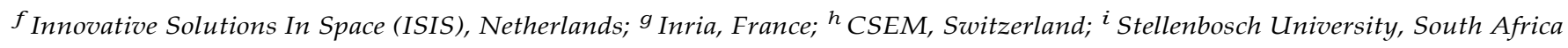

\begin{abstract}
This is the first of two companion papers that describe the development of the RemoveDEBRIS; mission. This first article focusses on the mission design and hardware development up to the delivery of the spacecraft to the launch authority. The Secord article describes the in-orbit operations.

The European Commission funded RemoveDebris mission has been the world's first Active Debris Removal (ADR) missions to demonstrate, in orbit, some cost effective key technologies, including net and harpoon capture; and elements of the whole sequence of operations, like the vision-based navigation, ultimately planning to terminate the mission with the deployment of the dragsail to de-orbit the craft. The mission has utilized two $2 \mathrm{U}$ CubeSats as artificial debris targets released from the main $100 \mathrm{~kg}$ satellite, to demonstrate the various technologies.

This paper examines the design of the mission from initial concepts through to Manufacture, Assembly Integration and Testing of the payloads, up to launch, and apart from a general consideration of the mission, will focus on the elements of design and testing that differ from a conventional mission.
\end{abstract}

Keywords: space debris, debris removal, Active Debris Removal, ADR, deorbiting, net, harpoon, vision-based navigation, dragsail

\section{INTRODUCTION}

REMOVEDEBRIS has been the first low cost mission to perform key active debris removal (ADR) technology demonstrations including the use of a net, a harpoon, vision-based navigation and a Dragsail in a realistic space operational environment. For the purposes of the mission two CubeSats ware ejected and then used as targets instead of real space debris.

\footnotetext{
*Corresponding Author. Tel.: +44 (0)1483 684317

Email addresses: g.aglietti@surrey.ac.uk

URL: www.surrey.ac.uk/ssc/

${ }^{1}$ Consortium Dissemination Manager, Research Fellow II

${ }^{2}$ Principal Investigator, Professor of Spacecraft Engineering
}

This paper examines the design of the mission from initial concepts through to manufacture, AIT, testing and up to launch. The mission operations from launch to the various inorbit demonstrations are described in the companion article [1]. Apart from a general consideration of the mission design, only elements of testing that differ from a conventional mission will be examined.

For more details about the mission design evolution refer to: website [2], first major mission paper [3], mission analysis [4], functional and environmental experimental results [5], [6], former mission and launch update [7]. 


\section{I.1. Landscape - state of the art}

One of the most active in the field of debris removal is the European Space Agency (ESA). ESA has produced a range of CleanSpace roadmaps, two of which focus on (a) space debris mitigation and (b) technologies for space debris remediation. A main part of these roadmaps is e.Deorbit, a programme spanning a host of phase studies examining removing a large ESA-owned object from space [8] [9]. This initiative started with ESA's Service Orientated Active Debris Removal (SOADR) Phase 0 study involving the analysis of a mission that could remove very heavy debris from orbit examining both the technical challenges and the business aspects of multiple ADR missions [10] [11]. Progressing on, ESA has also now completed Phase A (feasibility) and Phase B1 (PDR) studies [12] [13], with now several more mature designs now available. ESA's Satellite Servicing Building Blocks (SSBB) study originally examined remote maintenance of geostationary telecommunications satellites using a robotic arm [14]. The French space agency, CNES, is also widely involved in debris removal and has funded studies such as OTV which traded-off different ADR mission scenarios [15]. DLR's (German space agency) DEOS (Deutsche Orbital Servicing Mission) went as far in design as PDR level and aimed to rendezvous with a noncooperative and tumbling spacecraft by means of a robotic manipulator system accommodated on a servicing satellite [16].

Regarding the development of capture technologies, there are several on-going efforts. Airbus capture designs include the robotic arm, net [17], and harpoon demonstrators for use in space [18]. The net, in particular, is considered by some studies to be the most robust method for debris removal, requiring the least knowledge about the target object [10]. The First European System for Active Debris Removal with Nets (ADR1EN) is testing net technologies on the ground with the aim of commercialising later on. A host of other capture technologies have also been proposed including: ionbeam shepherd [19], gecko adhesives and polyurethane foam [20] [21]. Aviospace have been involved with some ADR studies such as the Capture and De-orbiting Technologies (CADET) study which is examining attitude estimation and non-cooperative approach using a visual and infra-red system [22] and the Heavy Active Debris Removal (HADR) study that examined trade-offs for different ADR technologies, especially including flexible link capture systems [23]

Although recently there have been advances in relative space navigation, the complex application of fully uncooperative rendezvous for debris removal has not yet been attempted. Vision-Based relative Navigation (VBN) systems, which would be necessary for future debris removal missions are currently being developed and will be demonstrated on RemoveDebris [24] [25] [26]. Other recent research specifically related to VBN for debris removal includes: TU Dresden [27], Thales [28], Jena-Optronik [29].

A range of de-orbitation technologies have been proposed previously but few have had in-flight testing. Research includes: Dragsails (InflateSail, DeOrbitSail) [30] [31],, TeSeR (which proposes an independent modular deorbitation module that attaches to the satellite before launch) [32], BETS propellantless deorbiting of space debris by bare electro- dynamic tethers (which proposes a tether-based removal system), solid rocket deorbitation (proposed D-ORBIT D-SAT mission) [33].

Regarding rendezvous in space, the Autonomous Transfer Vehicle (ATV) was one of the first times a spacecraft initiated and commenced a docking manoeuvre in space in a fully autonomous mode [34]. The Engineering Test Satellite VII 'KIKU-7' (ETS-VII) by JAXA in 1997 was one of the first missions to demonstrate robotic rendezvous using chaser and target satellites [35]. The AoLong-1 (ADRV) 'Roaming Dragon' satellite was also recently launched by CNSA (China National Space Administration) in 2016 in order to test target capture with a robotic arm; results are presently not available. Most recently JAXA's HTV-6 vehicle, which launched in early 2017, unsuccessfully attempted to deploy an electrodynamic tether under the Kounotori Integrated Tether Experiment (KITE) [36].

Upcoming missions to tackle debris removal include CleanSpace One by EPFL, which aims to use microsatellites with a grabber to demonstrate capture [37], [38]. The mission is still under design and launch is not foreseen for a few years. As mentioned previously, ESA's e.Deorbit will likely result in a large scale mission and is currently proposed for 2023. Of interest is Astroscale, aiming to launch a mission with thousands of 'impact sensors' to build up knowledge of the magnitude of small fragments [39] as well as testing a chaser for capture of a 'boy' target in their ELSA-d mission [40].

In this context, the RemoveDebris mission makes a significant contribution to the state of the art being the world's first Active Debris Removal (ADR) missions to demonstrate, in orbit, key future debris removal technologies and besides testing novel technologies (such as the net or harpoon) for the first time, has also set up an architectural design from which future missions could be based on.

The project has been carried out by a consortium of ten partners whose responsibilities are given in Table 1.

\section{I.2. Paper Structure}

Sections 2 to 4 focus on the mission design: concept / architecture, launch, operations. Sections 5 and 6 exam- 
ine the platform and CubeSats and deployers respectively. Sections 7 to 10 examine the individual payload design and testing. Finally, Section 11 concludes the paper and outlines key contributions to the field.

Table 1: RemoveDebris Consortium Partners. ${ }^{\dagger}$ vision-based navigation

\begin{tabular}{l|l} 
Partner & Responsibility \\
\hline $\begin{array}{l}\text { SSC (Surrey Space } \\
\text { Centre) }\end{array}$ & $\begin{array}{l}\text { Projectmanagement, Cube- } \\
\text { Sats, Dragsail, Harpoon Target } \\
\text { Assembly }\end{array}$ \\
\hline Ariane Group & Mission and systems technical lead \\
\hline Airbus Germany & Net \\
\hline Airbus France & $\begin{array}{l}\text { Overall VBN }{ }^{\dagger} \text { experiment } \\
\text { and algorithms }\end{array}$ \\
\hline Airbus UK & Harpoon \\
\hline SSTL & Platform technical lead, operations \\
\hline ISIS(InnovativeSolu- \\
tions in Space) & CubeSat deployers \\
\hline CSEM & LiDAR camera \\
\hline Inria & VBN algorithms \\
\hline Stellenbosch & CubeSat avionics \\
University &
\end{tabular}

\section{MISSION CONCEPT}

\section{II.1. High Level Architecture}

The RemoveDebris mission is based around a main chaser (called 'platform') from which the 'artificial debris' (the two CubeSats) are ejected, and where the main payloads are housed. There are 3 main experiments that utilize the two CubeSat targets, DS-1 and DS-2 these are: net, harpoon, vision-based navigation (VBN); a final 4th experiment is a dragsail for de-orbiting. The mission features are summarized in Table 2 .

\section{II.2. Scalability of Mission}

The degree of realism to which the on-board experiments represent full operational ADR scenarios, depends strongly on the future targets to be removed. Much research has shown that the removal of several heavier pieces of debris from space is one potential option [11]. As mentioned previously ESA is currently focusing on the removal of a larger piece of space debris through the CleanSpace initiative [41]. The heavier debris considered in this scenario is several tonnes in size. From a scalability perspective, the net and harpoon demonstrated on RemoveDebris are smaller scaled down versions of those considered for e.Deorbit. This is because the same Airbus DS teams that are working on the e.Deorbit scenario are present on this RemoveDebris mission. The net system is virtually the same system but smaller. The core difference in the harpoon system is that the RemoveDebris version uses a cold gas generator to provide the pressure to fire the harpoon. However, it is to be noted that core the harpoon system, projectile, and target material is the same for both scenarios.

Regarding the representativeness of firing a harpoon on to a target plate as in the RemoveDebris mission, which will be deployed at $1.5 \mathrm{~m}$ from the platform, as opposed to an uncooperative target, the experimental setup is still extremely valuable. Firstly, this will be the first firing of a harpoon system in space and will elevate the system's TRL. The complexities of firing a harpoon on to an uncooperative target are not to be underestimated. Firstly a chaser would have to rendezvous and match attitudes with target. Then the chaser would have to very precisely point and fire the harpoon (initial estimates require an accuracy of greater than 1.5 degrees).

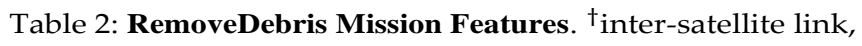
* payload interface unit

\begin{tabular}{|c|c|c|}
\hline Platform & $\begin{array}{l}\text { Structure } \\
\text { AOCS } \\
\text { Comms Power } \\
\text { Avionics }\end{array}$ & 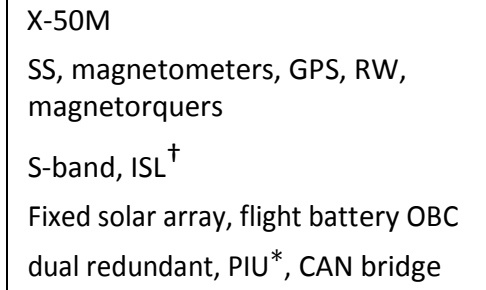 \\
\hline Targets & $\begin{array}{l}\text { DS-1 Cube-Sat } \\
\text { (net) } \\
\text { DS-2 Cube- Sat } \\
\text { (VBN) } \\
\text { Deployable } \\
\text { target } \\
\text { (harpoon) }\end{array}$ & $\begin{array}{l}1 \times \text { passive CubeSat, inflatable } \\
\text { structure, low-speed } 5 \mathrm{~cm} / \mathrm{s} \text { deployer } \\
1 \times \text { active CubeSat with AOCS, GPS, } \\
\mathrm{ISL} \text {, deploy- able solar panels, low- } \\
\text { speed } \mathrm{cm} / \mathrm{s} \text { deployer } \\
\text { OSS deployable boom, fixed target } \\
\text { plate }\end{array}$ \\
\hline Payloads & $\begin{array}{l}\text { Net } \\
\text { Harpoon } \\
\text { VBN } \\
\text { Dragsail } \\
\text { Supervision } \\
\text { cameras }\end{array}$ & $\begin{array}{l}1 \times \text { netfiredonDS-1 in open-loop at } \\
7 \mathrm{~m} \\
1 \text { xharpoon fired ontarget plate } \\
\text { at } 1.5 \mathrm{~m} \\
\text { LiDAR, 2-D camera pointing at DS- } 2 \text { for } \\
\text { analysis from } 0 \text { to } 1800 \mathrm{~m} \\
\text { Dragsail deployable to } 9 \mathrm{~m} 2 \text { on } \\
\text { platform } \\
\text { xdual-redundantcameras recording } \\
\text { experiments }\end{array}$ \\
\hline
\end{tabular}

Both of these require a precision closed loop attitude control system on-board the chaser. Apart from the complexities of the chaser AOCS system, firing a tethered harpoon on to an independent target also can result in a 'bounce-back collision', where the resulting target and harpoon return to hit the chaser. This presents very high risk to the mission and the current experimental setup provides a good compromise on this mission, which is also acceptable to the licensing authorities. 


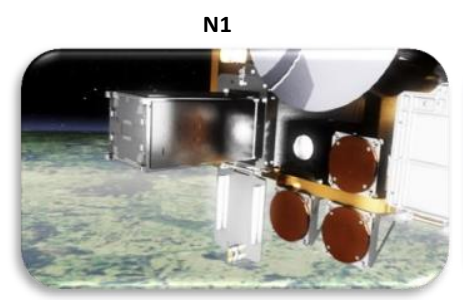

V1

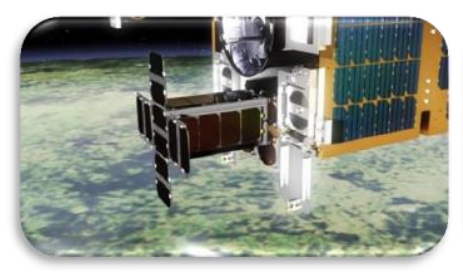

H1

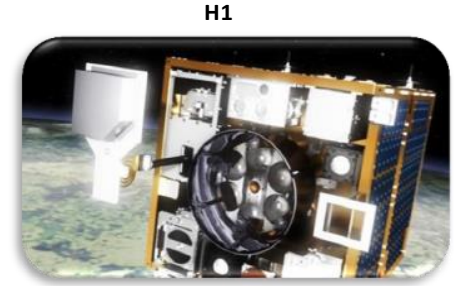

D1

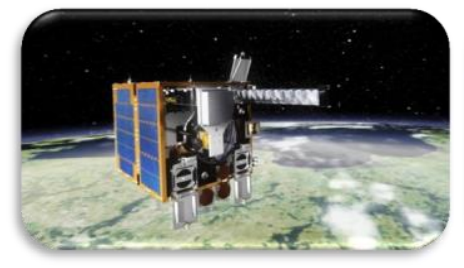

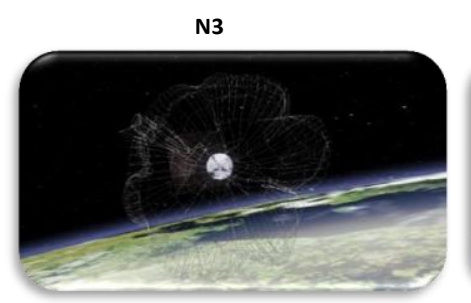

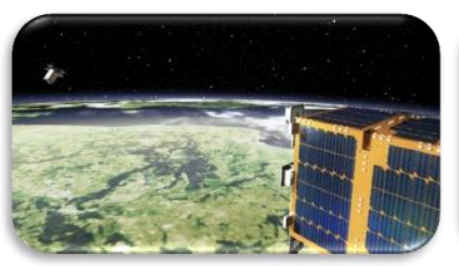

H2

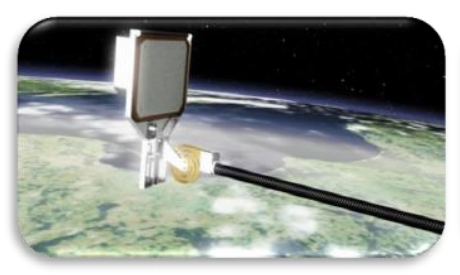

D2

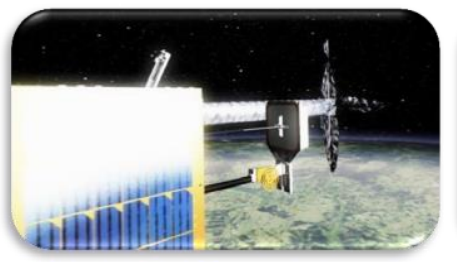

V3
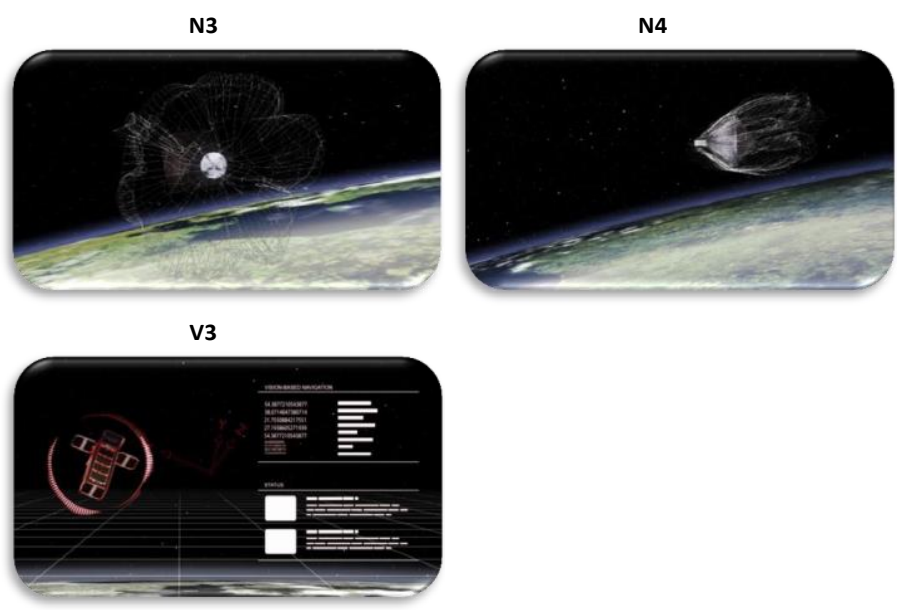

H3

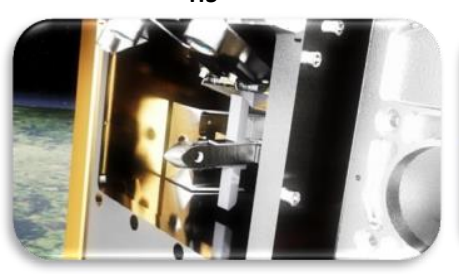

D3

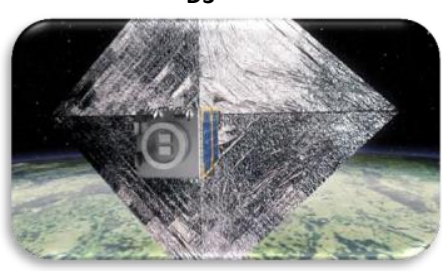

Figure 1: Demonstration Sequence. This figure shows the demonstration sequences for the net (N1 to N4), VBN (V1 to V3), harpoon (H1 to H4) and dragsail (D1 to D3). Note: visualisation is only an approximation of the mission - subsystems may be positioned differently or have cosmetic differences, compared with flight model.

A final note is on the use of CubeSats as artificial debris targets. A prime advantage of doing this (apart from the fact that if real debris was used, the chaser would have to move itself to the debris and rendezvous therefore requiring an onboard propulsion system) is that this avoids any legal issues with targeting, capturing or deorbiting debris that is legally owned by other entities, which would require further permission.

\section{II.3. Overview of In-orbit Demonstrations}

This section details the several in-orbit demonstrations in the mission. The four experiments are performed sequentially; with data from each being downloaded before the commencement of the next experiment. There is expected to be 6 month of mission operations. The four core mission demonstrations are shown in Figure $11 \mathrm{~N}$ and are described in details in section IV, proposed operations.

\section{LAUNCH PLANNING}

\section{III.1. Orbit and Launch Selection}

The RemoveDebris platform was developed to be launched accommodated within a Dragon resupply mission (SpaceX) to the ISS and from here released in space. There were various practical reasons for the selection of the ISS: (a) Nanoracks was expanding its business line to accommodate the launch of larger spacecraft from the ISS, as opposed to just CubeSats, which now presents a competitive launch option; (b) the altitude of the ISS is low enough to guarantee that there will be no violation of 25 year deorbitation laws (see deorbit times section) which provides more confidence to the UK space agency (the regulatory body) in licensing the mission.

Hence, the mission baseline orbit is the ISS orbit (51.6 $)$ and approximately a $400 \mathrm{~km}$ altitude, circular at the beginning.

For further information about the mission trade-offs see [3]. 


\section{III.2. Planned Launch Sequence}

The launch sequence for the RemoveDebris mission is an unconventional one. The solution uses NanoRacks as a supply agent to launch the final flight platform to the International Space Station (ISS) abroad a SpaceX Dragon capsule. The sequence of operations can be seen in Figure 2. Before launch (1), the cargo bag is loaded into the Dragon capsule as cargo and strapped down. After the cargo is launched to the ISS (2), the clam shell and outer protective panels are unpacked by astronauts, which install the platform on to the Japanese experiment module (JEM) air lock table (3). The air lock then depresses and the slide table extends. The platform is grappled by the JRMS, a robotic arm system (4). Finally, the robotic arm positions and releases the platform into space (5), where commissioning and main operations of the mission can commence. Naturally, the ejection trajectory ensures that the satellite will not intersect the ISS orbit at a later time.

\section{III.3. Overview of Regulatory Considerations}

The mission aims to comply with legal requirements for deorbiting including that (as mentioned before) objects placed in LEO (low Earth orbit) should naturally deorbit within 25 years, a key requirement of the UK Outer Space Act (OSA, 1986) and the French Space Operations Act (2008).

Table 3: RemoveDEBRIS Deorbit Times. From STELA (in 2016, from $400 \mathrm{~km}$ ).

\begin{tabular}{l|l} 
Object & NominalOrbit Lifetime(yrs) \\
\hline Platform (RemoveSAT) & 2 \\
DS-1 (Net) & 0.4 \\
DS-2 (VBN) & 0.5 \\
Net (alone) & 0.5 \\
Harpoon (alone) & 2
\end{tabular}

Various packages have been used to calculate the deorbit time for all objects placed in space including ESA's DRAMA (debris risk assessment and mitigation analysis) and CNES's STELA (semi-analytic tool for end of life analysis) [43]. In this research we present the results from STELA for each space object. Various interdisciplinary topics are involved in the evaluation of the orbital life- time, including solar activity prediction and its effect on the atmospheric density, solar radiation pressure and drag modelling, third body effects as well as complex gravity models implementation.

However, semi-analytical propagation techniques allow to evaluate the reentry duration in a reasonable computational time [44]. STELA has been validated by comparison to simulations based on fully numerical integration as well as real trajectories [46]. Table 3 summarizes the preliminary results obtained. The results show that the compliance to the 25 years rule is easily achieved for all the objects, even for the main platform when the drag sail is not deployed.

The use of the ISS scenario, launching to approximately $400 \mathrm{~km}$, provides greater confidence to licensing agencies as to the mission safety, as if there were any issues, all the items would de-orbit very quickly. [3] and [4] give more information about the orbital lifetime of the objects calculated using both STELA and DRAMA, specialist end-of-life tools. They show that the main platform deorbits within 2 years, even in case of the dragsail not deploying; smaller items, such as the CubeSats, de-orbit within a matter of months. Thus no further space debris is generated.

Launching to the ISS requires NASA safety reviews have to be passed. NASA impose certain constraints on the overall platform design to ensure safety to the astronauts on the ISS. As well as more common requirements, such as the platform not having sharp edges, several other requirements have introduced extra design effort in to the mission. These are detailed as follows. After ejection from the ISS, the main platform is inert for up to 30 minutes before booting on. This is to protect the ISS from interference, or in case of any issues. All batteries on the mission must have triple electrical inhibits and thermal run-away protection. This includes the main platform battery and the two batteries in the CubeSats. The CubeSats also can only turn on when three separate deployment switches are activated, which is only physically possible when the CubeSats have left their respective pods. Mechanically, all the payloads require an inhibit.

Significant effort has been extended to ensure astronaut safety. The harpoon can only fire with an 'arm and fire' sequential command sequence (which would of course require power to the system - which already has a triple electrical inhibit). Without this command, there is no way the cold gas generator (CGG), which propels the harpoon, could be powered, and thus no way in which the harpoon could fire. Furthermore, the safety door in front of the harpoon only opens before firing and must be manually commanded to be opened. In front of the safety door is the main target plate which presents another mechanical barrier. A final mechanical barrier is the Kapton box in front of the target plate which prevents possible fragments of debris escaping into space during the harpoon experiment. 


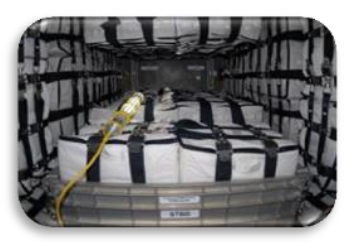

1

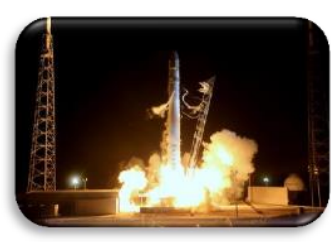

2

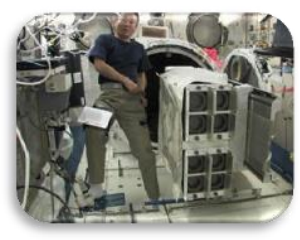

3

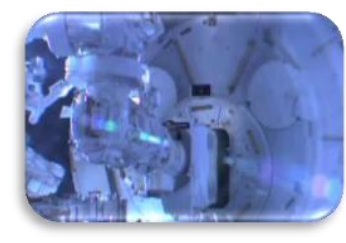

4

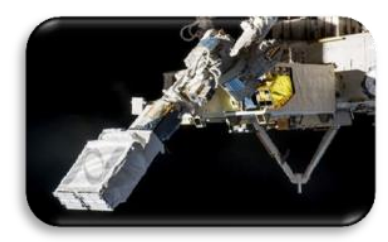

5

Figure 2: Launch Sequence. This figure shows the launch sequences for the mission to the International Space Station (ISS). Credit: SpaceX, NanoRacks, NASA [40].

\section{III.4. Shipping and Flight Preparations}

The RemoveDebris platform will be loaded on to the Dragon as cargo. The main platform is protected by a series of concentric encasements for shipping. Firstly cover panels screw into the platform structure and protect the solar panels. Secondly the panelled structure is placed within a clam shell. This clam shell is placed into a metal protective box and the box is put into the shipping container. On arrival at the launch facility, the platform is unpacked down to the clam shell and the clam shell is loaded into a cargo transfer bag (CTB) and then on to the Dragon capsule.

\section{PROPOSED OPERATIONS}

The proposed mission timing can be seen in Figure 4. The four core events are launch preparation, launch to the ISS, ejection from the ISS and mission demonstrations.

Figure 3 shows the mission space and ground segment for the proposed launch. Operations for the RemoveDEBRIS mission will be carried out from SSTL's Mission Operations Centre in Guildford. Company standard operations procedures will be used, which are compatible with the designed platform operational requirements and characteristics. Figure 4 is the mission timeline which shows the order in which experiments are to be performed. The net experiment is performed first, providing a target to help validate and calibrate the VBN system. The next experiments in order are VBN, harpoon and finally dragsail at the end of the mission.

\section{IV.1. Net Demonstration}

The net scenario is shown in Figure $1(\mathrm{~N})$ and is designed to help mature net capture technology in space. In this experiment, initially the first CubeSat (net), DS-1, is ejected by the platform at a low velocity $(5 \mathrm{~cm} / \mathrm{s})(\mathrm{N} 1)$. DS-1 proceeds to inflate an inflatable structure (N2) which, as well as acting as a deorbiting technology, provides a larger target area of $1 \mathrm{~m}$. A net from the platform is then ejected when the DS- 1 is at $7 \mathrm{~m}$ distance (N3). Once the net (now $5 \mathrm{~m}$ in size) hits the target (N4), deployment masses at the end of the net wrap around and entangle the target and motor driven winches reel in the neck of the net preventing re-opening of the net. The CubeSat is then left to deorbit at an accelerated rate due to the large surface area of the inflatable. During the net demonstration, two supervision cameras record images which are downloaded afterwards to ground to assess the success of the net demonstration.

The actual demonstration will start with checking the platform is ready to start the demonstration, and charging and turning on relevant platform services. Although the VBN demonstration comes after the net demonstration, the VBN requires calibration during the net demonstration and thus the full VBN image capture, transfer and download chain is performed to ensure the VBN is ready. The PIU (payload interface unit) on the platform is used to collect and process payload data. Part of the initial checks are that the supervision cameras have clear images - incorrect platform attitudes or poor lighting conditions (location in orbit) could mean images are obscured or too light or dark. There is therefore an opportunity to correct these before the demonstration begins.

On starting the main experiment the 2 platform supervision cameras activate and record the entire demonstration. At T0, the ISIPOD door opens releasing and translating the CubeSat into a locked position outside the ISIPOD. A timer cuts the CRS (CubeSat Release System) and the CubeSat is released. Shortly after, the DS-1 inflatable (via the CGGs) is inflated (Figure 1-N2), and the net is ejected to capture DS-1 (Figure 1-N3). The experiment closes with collection and download to Earth of the VBN and supervision cameras data. The net and DS-1 naturally de-orbit at a rapid rate due to the low altitude.

The main data collected in this experiment is the video of the experiment (from 3 sources). Various telemetry can also be acquired from the platform and the initial VBN experiment provides additional data sources.

\section{IV.2. VBN Demonstration}

The demonstration sequence planned for the VBN experiment is shown in Figure 1(V). In this experiment, the second CubeSat, DS-2, is ejected by the platform at very low velocity $(\sim 2 \mathrm{~cm} / \mathrm{s})$ out of the orbit plane (AoA: $110^{\circ}$, bank angle: $\left.100^{\circ}\right)(\mathrm{V} 1)$. The deployment direction is defined to comply with safety constraints and VBN demonstration needs (lightning, background, range). 


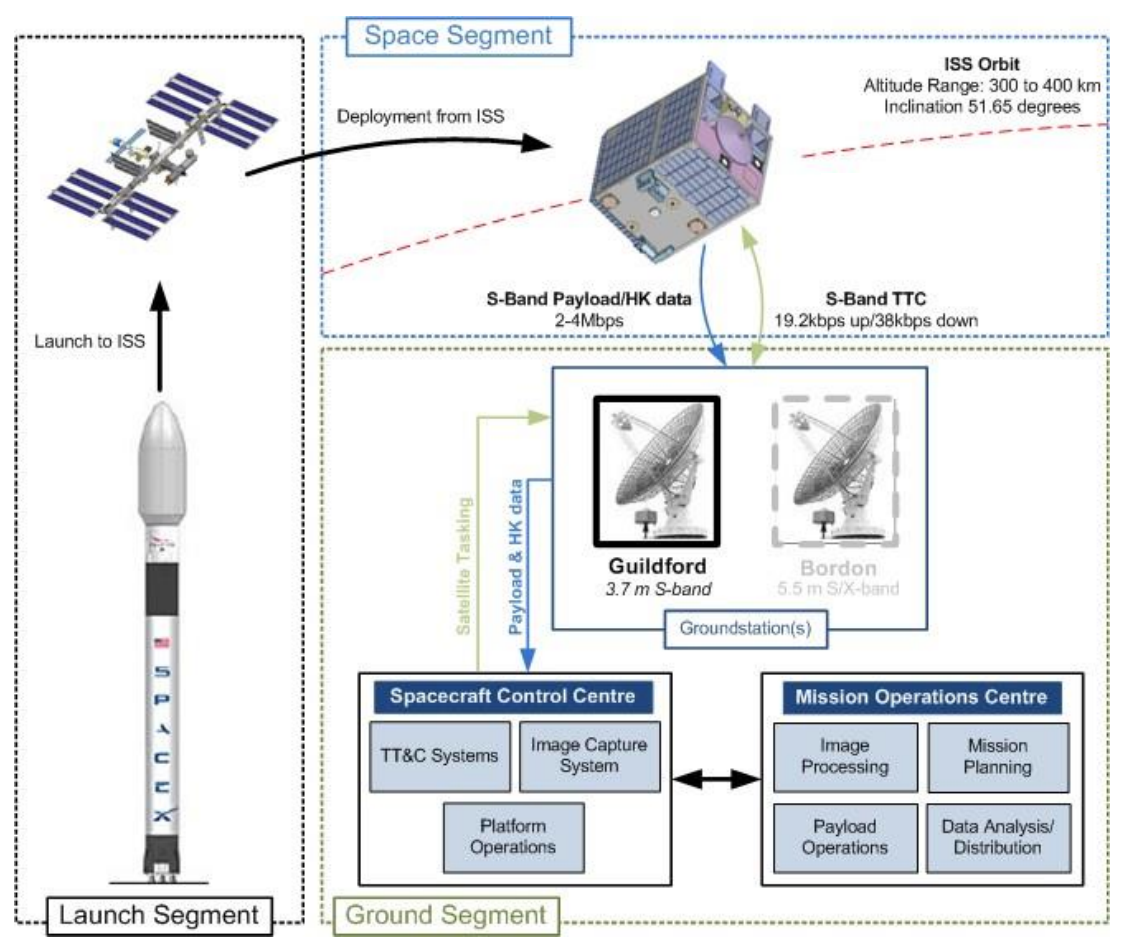

Figure 3: Overview of Mission Segments. This figure shows the three mission segments: launch, space, ground.

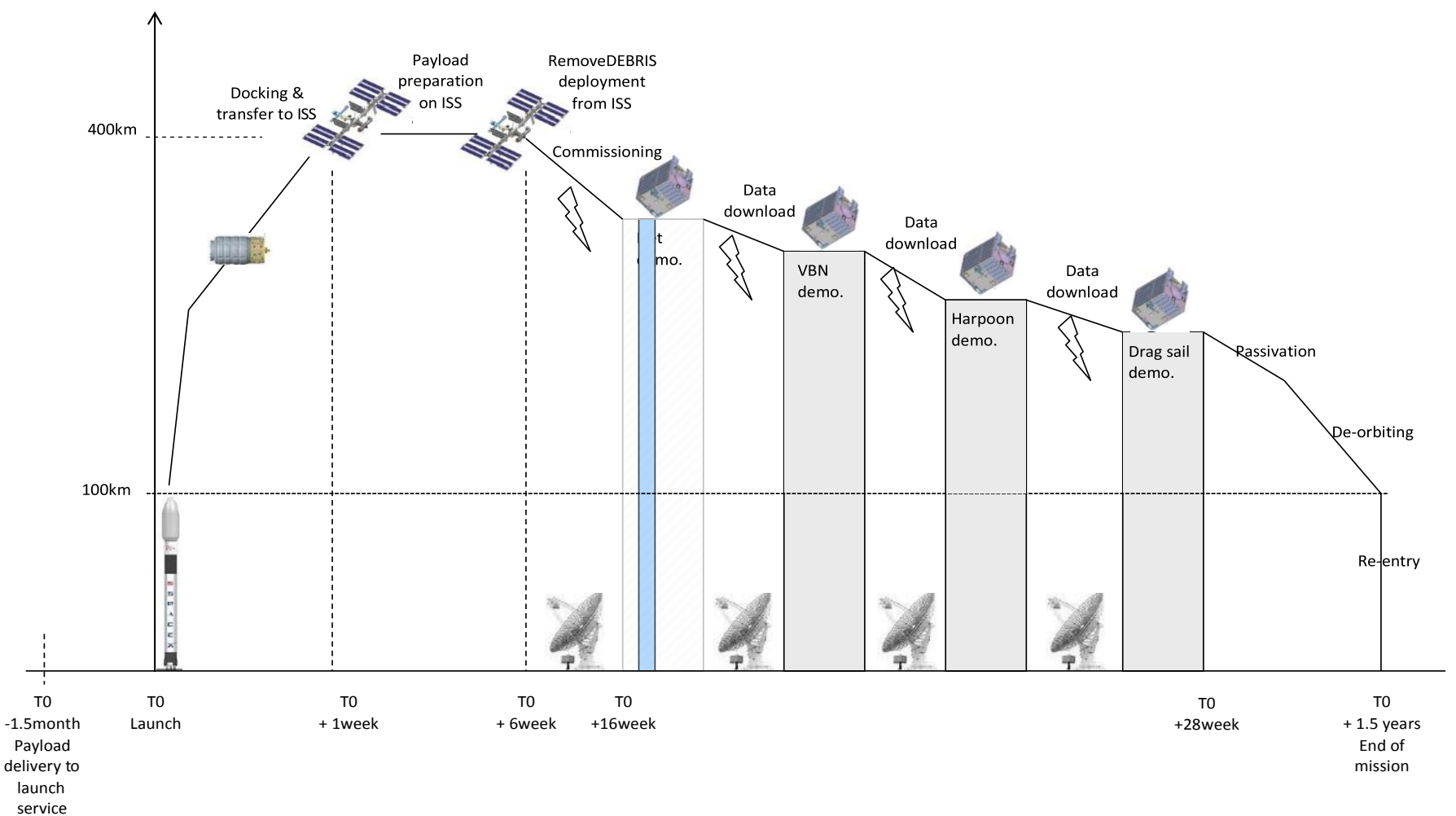

Figure 4: Proposed Mission Timeline. This figure shows the order in which experiments are performed, with very approximate altitudes for the experiments. All the capture experiments are planned to happen between $250 \mathrm{~km}$ and $350 \mathrm{~km}$. The exact timing (and altitudes) will depend on the operations sequence which is to be precisely defined. 
The cameras for the VBN system have been calibrated during the Net experiments. The DS-2 deployment (V2) direction enables to meet VBN objectives without need of platform boost. Platform attitude needs to be controlled in open loop only. Data, imagery and GPS data collected during VBN demonstration over few orbits are later postprocessed on ground (V3).

In practice the demonstration will starts with checking the platform is ready to start the demonstration, and charging and turning on relevant platform services. For clarity, there are 2 supervision cameras on the platform and $2 \mathrm{VBN}$ cameras $(3 \mathrm{~d}, 2 \mathrm{~d})$. Similar to the net demonstration, the VBN requires a calibration and test phase where the full VBN image capture, transfer and download chain is tested.

At T0, the ISIPOD door opens releasing and translating the CubeSat into a locked position outside the ISIPOD. Different to the net demonstration, DS-2 is given time here to flip open the solar panels, start its on-board services, acquire a GPS lock and initiate the inter-satellite link between DS-2 and the platform (ISL) (Figure 1 1-V1). The VBN cameras start recording from this point. After this is completed, a timer cuts the CRS (CubeSat Release System) and the CubeSat is released.

Entering the main VBN phase, both CubeSat and platform attitude are adjusted as required for the demonstration. The VBN and supervision cameras collect data on the platform and the data collected on DS-2 (including GPS data) is sent back via the ISL to the platform (Figure 1 1-V3). The experiment closes with collection and download to Earth of VBN system data, the supervision cameras data, and the acquired CubeSat data. DS-2 naturally de-orbits at a rapid rate due to the low altitude.

The data collected in this experiment includes: the video of the experiment (from 2 sources), the VBN video and system data (from the 2 cameras), the CubeSat data which includes attitude sensor data, GPS data and housekeeping data. The GPS data and attitude data is also available from the platform. These data sets will allow post-processing of data to validate the VBN concept and algorithms.

\section{IV.1. Harpoon Demonstration}

The sequence for the harpoon demonstration is shown in Figure $1(\mathrm{H})$, and it uses a deployable target that extends outwards from the platform which is used as a target for the harpoon (H1). The harpoon and the deployable target form the harpoon target assembly (HTA).

The deployable target is extended to its maximum extent at $1.5 \mathrm{~m}(\mathrm{H} 2)$ where the end target plate is $10 \times 10 \mathrm{~cm}$. At that point the harpoon fires $(\mathrm{H} 3)$ and captures the target plate (H4). The harpoon is designed with a flip-out locking mechanism that prevents the tether from pulling out of the target. As for net and harpoon demonstrations, success will be assessed by the images collected by the 2 supervision cameras up to $100 \mathrm{fps}$.

As usual, the demonstration starts with checking the platform is ready to start the demonstration, and turning on relevant platform services. In the first phase, the target boom must be extended (Figure 1-H1), which involves cutting the frangibolt holding the target in place and deploying the boom. This phase is recorded. As per the other demonstrations, the platform needs to be re-pointed into the correct direction, the VBN must be calibrated and the supervision camera images checked ready for the main experiment.

In the main part of the demonstration, the platform services are re-enabled ready for the firing. At T0, the harpoon payload service is turned on (this is not the point at which the harpoon fires). Shortly after the harpoon protection cover is released (Figure 1-H3), recording is started and the 2 CGGs (cold gas generators) that fire the harpoon are activated. The harpoon aims to impact the target plate (Figure 1-H4).

The experiment closes with collection and download to Earth of VBN system data and the supervision cameras data. Before finishing the demonstration, the harpoon is retracted slightly (which is also recorded).

The main data collected in this experiment is the video of the experiment (from 2 sources). Various telemetry can also be acquired from the platform and the initial VBN experiment provides additional data sources. A thermal sensor is also embedded in the harpoon target assembly.

\section{IV.2. Dragsail Demonstration}

The final experiment is the dragsail shown in Figure 1(D), which is deployed at the end of the mission to de-orbit the main chaser. The dragsail payload inflates a $1.0 \mathrm{~m}$ inflatable mast (D1) that moves the main sail away from platform. The $10 \mathrm{~m} 2$ sail is then drawn out by the unfurling of 4 carbon fibre booms (D2), until the sail is fully deployed (D3).

The demonstration starts like the other 3 to check whether the platform and payloads are in a suitable position to start the demonstration. The supervision cameras are activated and the dragsail power switches are activated at T0 (this is not the point at which the dragsail starts deployment). Shortly after the dragsail burnwire is cut to enable the mast to deploy, the boom venting valve is closed (see [4] for more information), and the 2 CGGs are activated to inflate the mast. After this, the deployment motors are activated to unfurl the sail and carbon fibre booms. The experiment closes with the download of supervision camera data to Earth. After the dragsail is deployed, the platform will deorbit at an accelerated rate. Due to the size of the sail, the platform does not guarantee unhindered communication or full power integrity (due to potential overlap of solar 
panels) after deployment; assessment of these is part of the demonstration.

Various telemetry can also be acquired from the platform. In particular, the influence of the deployed dragsail on the platform can be assessed through attitude (and generic AOCS) data, power data and communications systems data.

The platform de-orbit trajectory can be tracked from the ground and this can be compared with theoretical simulations of the de-orbit rate without a sail.
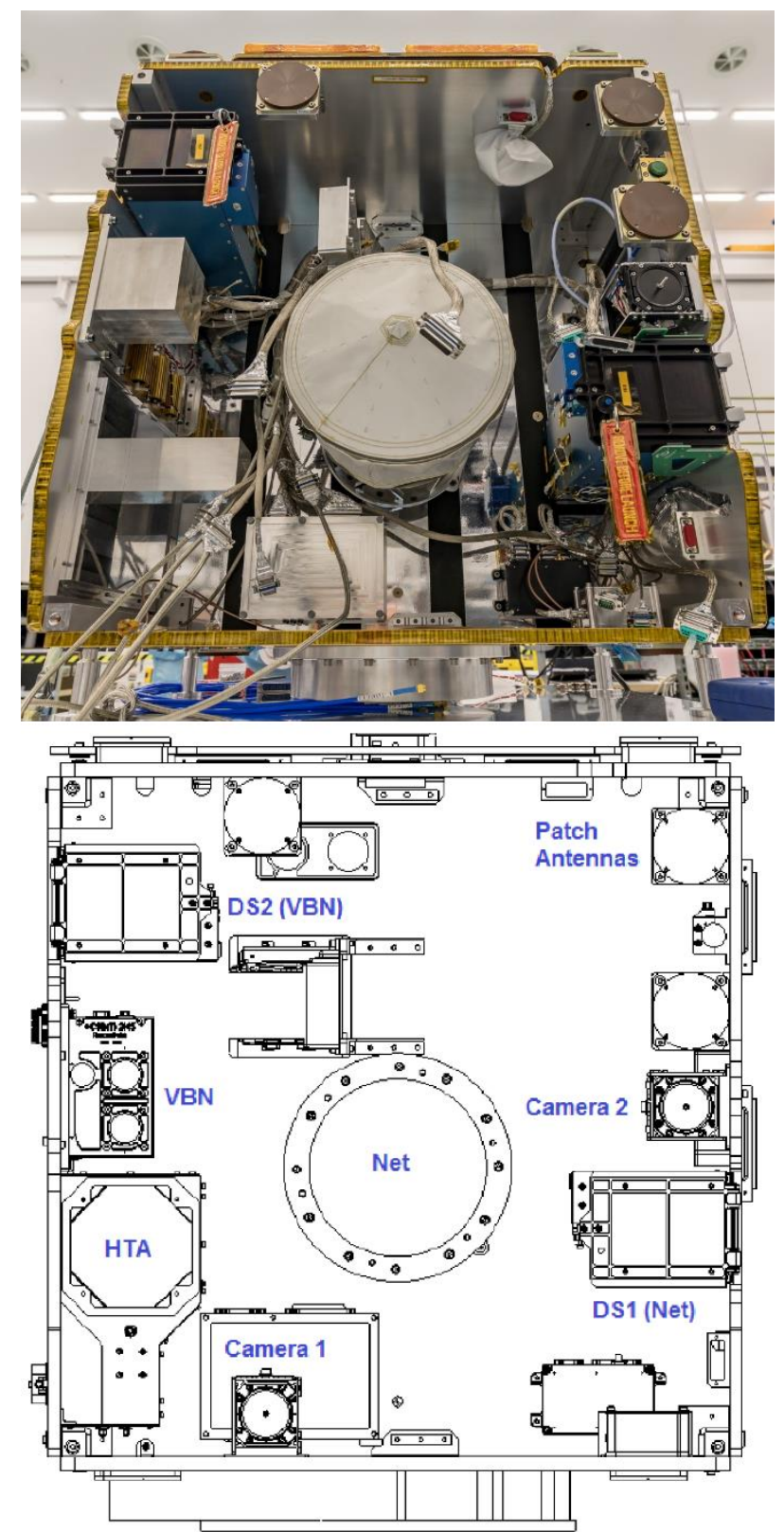

Figure 5: Platform - Payload Face. This figure shows the platform from the payload face. Top: platform under integration showing: 2 CubeSat deployers, net, 1 camera, patch antennas. Mass dummies integrated for the HTA and VBN subsystems. Bottom: CAD model view of the same face

\section{PLATFORM}

The platform, in the SSTL AIT hall, can be seen in Figure 5. Examining this figure, the centre of the platform houses the net to minimise any torque (and AOCS disturbances) to the platform when ejecting the net. DS-1 and its associated ISIPOD, used in the net demonstration, can be seen in the bottom right. The VBN system (not mounted in photo) is shown on the left-hand side with its corresponding DS-2 and ISIPOD directly above it. The HTA system is in the bottom left corner. Also visible are the supervision cameras and the patch antennas. Note that the dragsail has been reallocated to the back panel from former designs in [4].

Regarding subsystems, visible in Figure 6, the platform is based on the commercial X50 line and utilises internally developed avionics systems under the Fireworks programme [2]. In order to minimise mass the satellite structure is manufactured using honeycomb panels with aluminium face sheets. The core avionics, as listed in Table 2, take the form of a series of avionics cards that slot into card frames. The cards include: communications (sband), power distribution (PMAD), power charging, OBCs, PIUs (interface units for the payloads), AOCS and navigation. The main flight battery is also shown, along with the separate reaction wheel (two planar, two nonplanar). The magnetometers along each axis are not marked on the diagram, but are present in the back section.

Regarding testing, once the payloads and satellite modules were delivered and accepted into the assembly, integration, and test (AIT) facility the satellite underwent a conventional environmental test (EVT) campaign comprising of: EMC testing, mass property measurements, launch box integration and strip down, vibration testing, external inspections, spacecraft functional tests, thermal vacuum testing, integration of flight battery and some flight payloads, EVT results review. The EVT campaign verified and validated the spacecraft and tested it as a whole system. At various stages during the test campaign the satellite underwent system level functional tests to ensure the system continues to operate as expected.

\section{CUBESATS AND DEPLOYERS}

This section will focus on the two $2 \mathrm{U}$ CubeSats (each $100100227 \mathrm{~mm}$ ), used for the net and VBN demonstrations. The CubeSats are ejected from the platform then used as targets instead of real space debris, which is an important step towards a fully operational ADR mission. The CubeSats and deployers are each tested in their own framework first, before coming together for final integration and testing. Eventually the combined 
deployer with Cube- Sat inside is sent to the platform for payload integration and final testing. In both demonstrations the ejection speed is carefully controlled and the sequence of ejection can be seen in Figure 7 . Initially, the CubeSats sit inside their pods (ISIPODs). When the CubeSats are pushed out of the pod by a spring, they click into place at the end of the pod. The CubeSat Release System (CRS) is a burnwire that holds the CubeSat to the end of the pod. When burnt, microsprings push the CubeSats away from the pod (and platform) at a specific velocity adjusted through spring tuning.

Further information about the CubeSat design and operation can be found in [30], [46], [47].
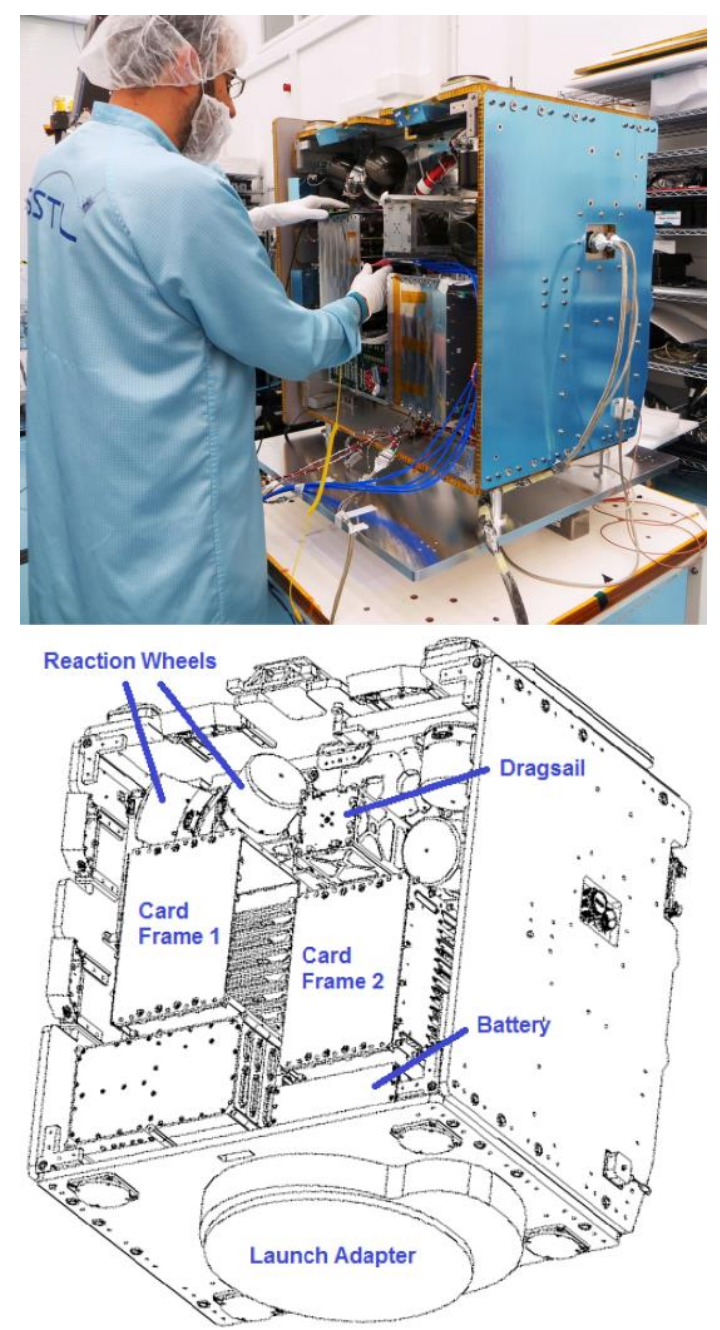

Figure 6: Platform - Back Face. This figure shows the platform from the back face. Top: platform under integration. Bottom: CAD model view of the same face.

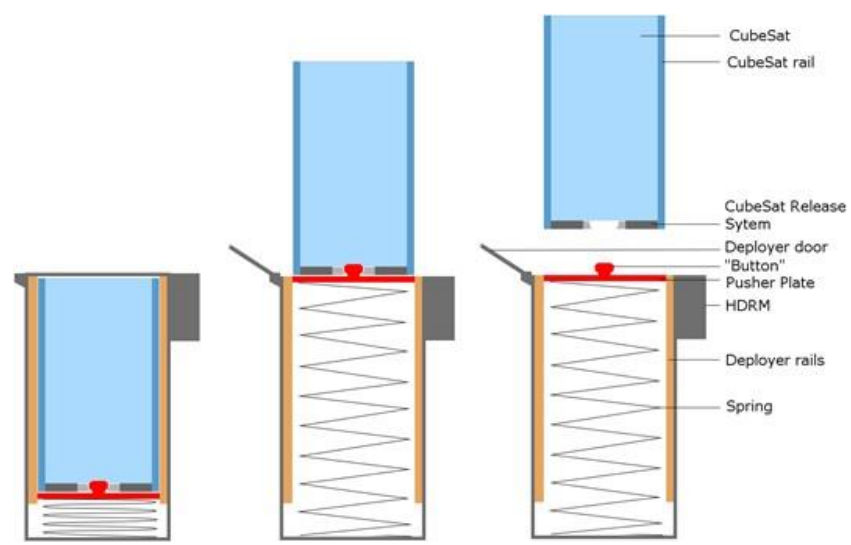

Figure 7: Mechanism of CubeSat Ejection.

The avionics boards, which are relatively uncomplex in the case of DS-1, are tested at a payload level before integration.

\section{VI.1. DS-1: NetCubeSat}

In the DS-1 CubeSat, the bottom half has the avionics and the top half has the inflatable structure, which inflates shortly after the CubeSat is released from the platform in order to provide a small demonstration of inflatable technology and to provide a larger target area for the net to capture. The DS-1 flight model can be seen in Figure 8 with the inflation side at the top. Two key functional testing regimes have focused on the testing of the aforementioned CRS and the inflatable system.

\section{VI.2. DS-2: VBN CubeSat}

In the VBN experiment, the VBN payload on the platform will inspect the VBN CubeSat, DS-2, during a series of manoeuvres at a range of distances and in different light conditions dependent on the orbit. The CubeSat, DS-2, can be seen in Figure 8.

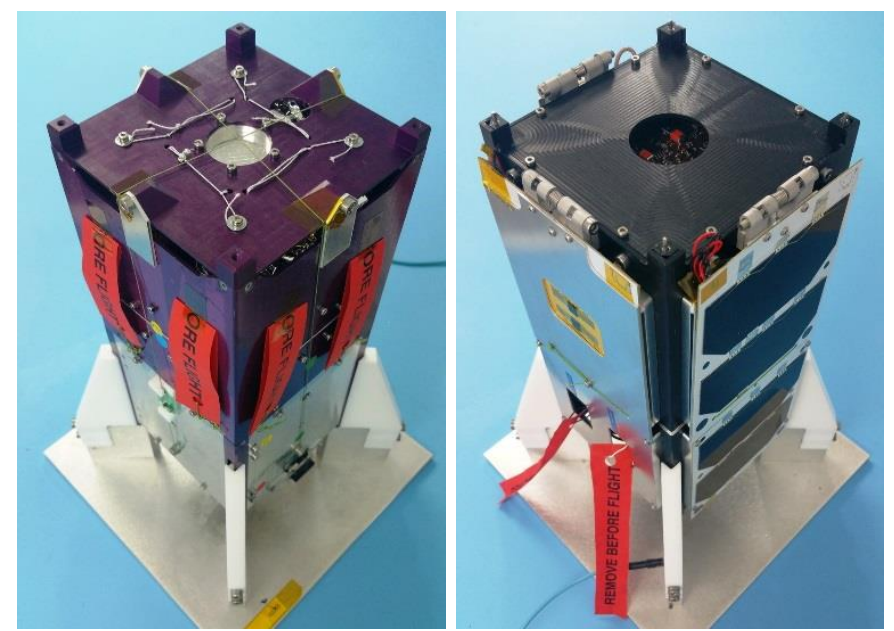

Figure 8: Flight Models (FM): DS-1 (left) and DS-2 (right). 
The avionics on-board include: the GPS board, 3 OBC boards which contain full 3-axis (3-DoF) attitude control, the EPS board, the burnwire board, an ISL (inter-satellite link) board, the camera board, and solar cells. Key functional testing focused on the testing of the: CRS, GPS and ADCS suite, ISL transmission chain. In addition, an extensive process of systems integration testing was performed to ensure hardware and software compatibility.

\section{VI.3. Functional Testing - Flight Software and Controls}

The CubeSat avionics are based on the QB50 avionics developed by Stellenbosch University and the Surrey Space Centre [48]. Verification requires several functional tests to be undertaken: sensor orientation checks, controller gain tuning, GPS start-up and lock acquisition tests. Since the QB50 mission, there have been updates to the software suite and ground control software which is used throughout Surrey Space Centre missions (on RemoveDebris the ground station segment is only used for remote testing, not in-flight, as there is no CubeSat to ground communications). [49] gives further details about the integrated flight and ground software framework and associated testing.

\section{VI.4. Functional Testing - ISL System End-to-end}

Figure 9. shows a hardware-in-loop System End-toEnd Test SEET (payload level), using the DS-2 CubeSat to photograph a simu- lated image of the platform (replicating its view in space), and transmit camera and sensor data back over the inter- satellite link (ISL) in real-time to a simulated platform. The CubeSat DS-2 was placed on a surface and rolled

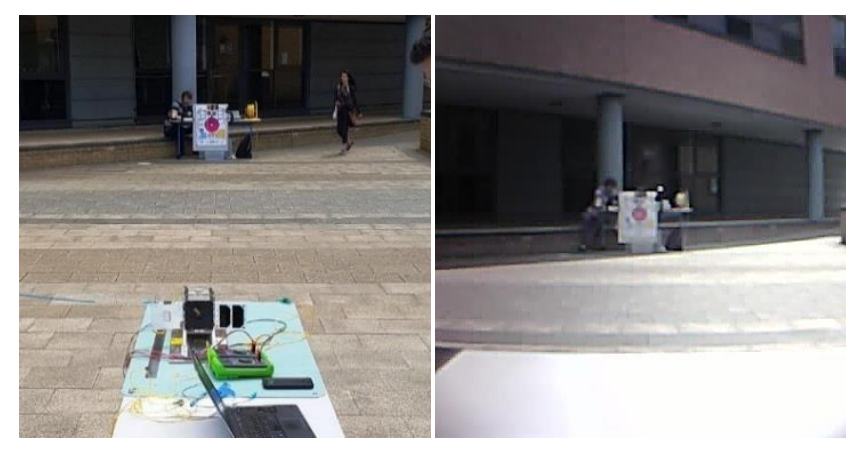

Figure 9: FM DS-2 and ISL SEET. Full end-to-end DS-2 CubeSat (containing camera, sensors and inter-satellite link) test transmitting data back to a simulated platform. Left: test setup with CubeSat shown on surface. Right: view from CubeSat camera (as transmitted back to the platform).

backwards at the same speed as CubeSat ejection on the actual mission. The data collection rate was the same as on the actual mission. The test shows the type of images and nature of data expected from these initial stages of the
DS-1 demonstration. In addition to this HILS test, the ISL link underwent independent RF range testing (which showed good performance even beyond the required $400 \mathrm{~m}$ range requirement) and a 90 hour soak test to burn in the components.

\section{VI.5. Functional Testing - Inflatable Structure}

The inflatable section of DS-1 contains: the central inflation connector system, a cold gas generator (CGG) which is the inflation source, a solenoid valve. Figure 10 shows the flight model (FM) packaged inflation system both with and without sail material. The transparent side panels are only for assembly and functional test purposes; in readiness for flight these panels are replaced with metal ones. The SEET (payload level) for DS-1 is shown in Figure 11. The CubeSat showed correct and full inflation, with gravity compensation, before it was repacked for transportation to the integration hall. Ref [50] gives further details on the inflatable design and testing methodology.
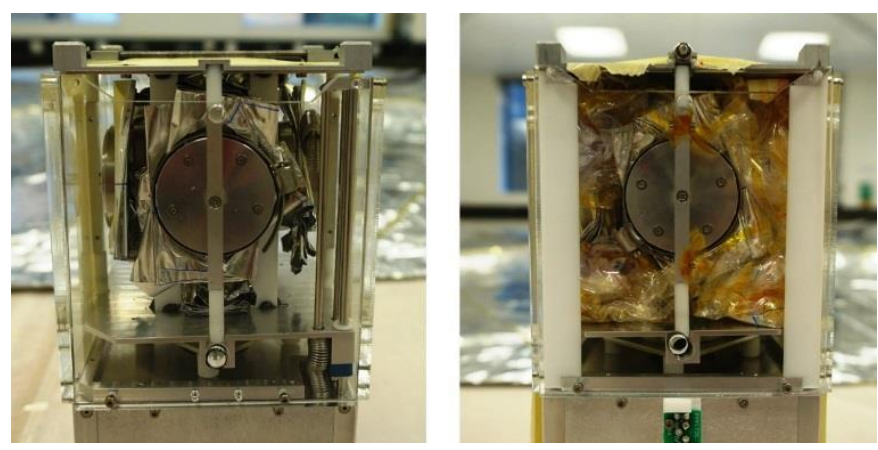

Figure 10: DS-1: Packaged Inflation Module.

VI.6. Functional Testing - Deployer and CRS

ISIPOD deployers and CRS are functionally tested separately including functional testing at cold and hot temperatures as well as characterisation of the delays due to ISIPOD hold-down and release mechanism (HDRM) and CRS actuations.

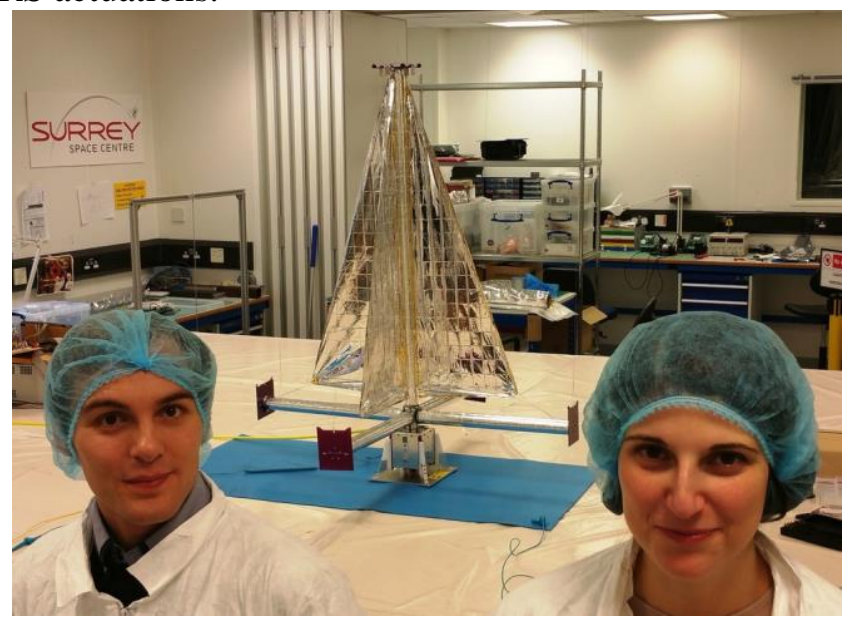

Figure 11: DS-1: FM CubeSat SEET Inflation. This figure shows the DS-1 CubeSat under a final deployment test as part of the SEET. 
The CRS is in charge of providing the accurate deployment velocity which is critical for the success of the DS-1 and DS-2 experiments. For the tuning of the CRS release springs, the release elements are fine-tuned based on the analytical results and the qualification and characterization information.

Finally once the CRS is integrated on the CubeSat and this on the deployer the deployment velocity and direction are measured. A long pendulum setup together with a high speed camera are used to perform the measurement (see Figure 12). The data is postprocessed via specific image software to retrieve the key information (see Figure 13) and that the deployment is within the bounds of the requirements.

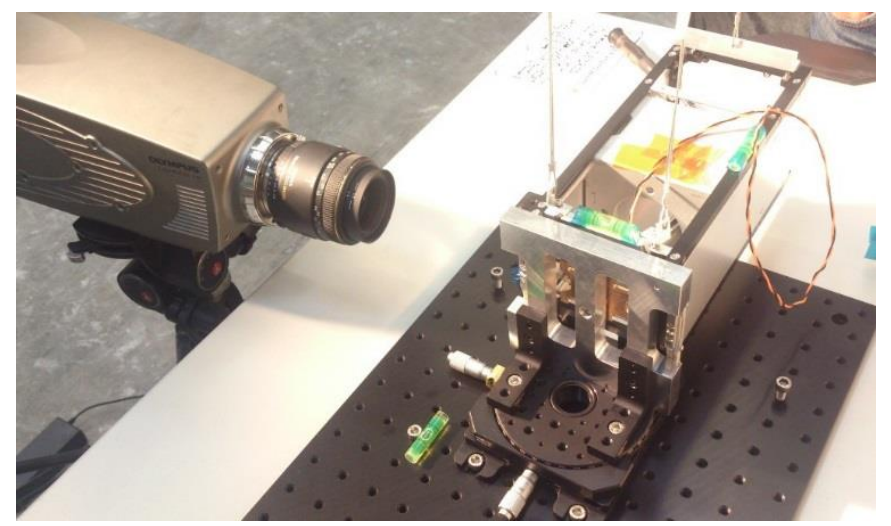

Figure 12: Deployer and CRS: Detail of the Velocity Testing Setup. 2U CubeSat suspended on pendulum and high speed camera.

\section{VI.7. Environmental Testing}

The functional tests described previously are undertaken throughout the environmental campaign. Both CubeSats and deployers initially underwent a range of individual en- vironmental testing including mechanical (vibration) and thermal. After the CubeSat and deployer are brought to- gether and the deployer spring tuning is done, the combined payload progresses through a further thermal cycling test (with CRS release) and a functional system end to end test (SEET) under vacuum. A final combined acceptance vibration test is performed before delivery to the platform for integration.

Both ISIPOD and CRS were subjected and successfully passed the qualification vibe and thermal tests according to ISIS general levels that cover all the RemoveDebris loads profiles. In addition both ISIPOD and CRS are subjected to shock measurement in order to measure the shock environment created on the CubeSat due to the different shock events during operations

\section{VI.8. Flight Preparations}

After the flight preparation and successful test campaign of the ISIPODs (RemoveDebris deployers), they were brought to SSTL facilities in order to perform the integration in the RemoveDebris platform together with the other payloads and subsystems. The CubeSat Release Systems (CRS) is the system in charge of ensuring the CubeSats are released from the platform with the required velocity. After the fine tuning of the CRS flight models together with the actual flight models of the CubeSats they were also ready to be brought for the final integration.

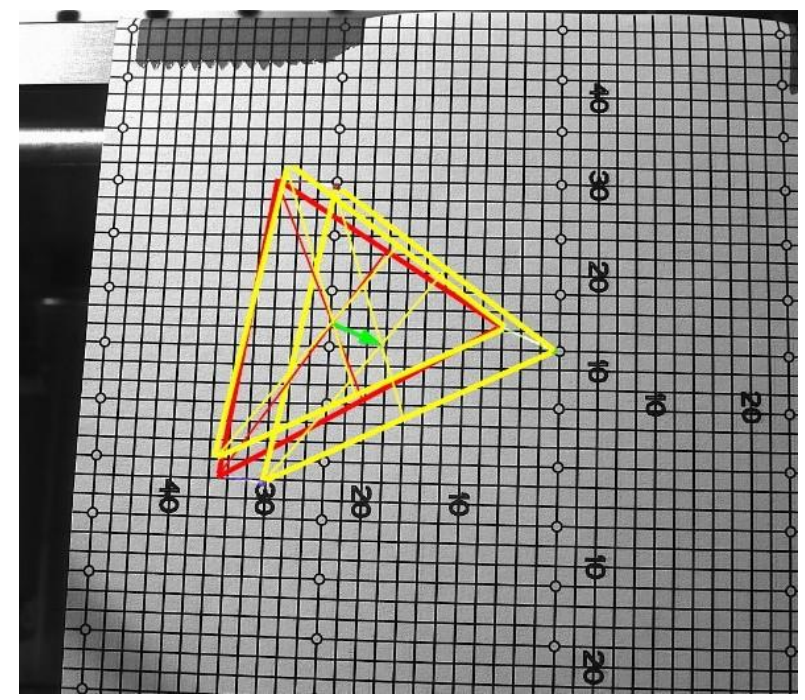

Figure 13: Deployer and CRS: Methodology for the post processing to retrieve ejection performance.
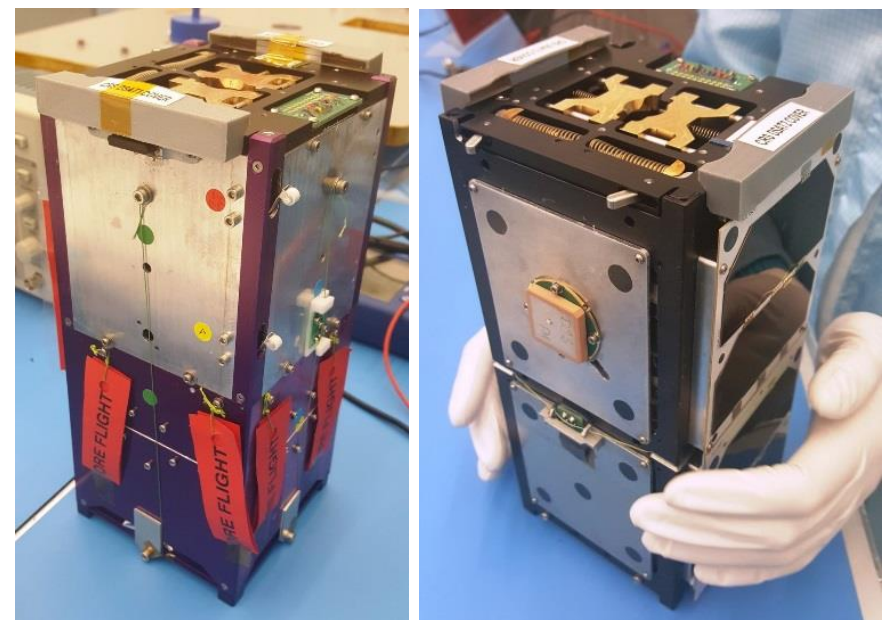

Figure 14: CRS insertion on to CubeSats. This figure shows DS-1 (left) and DS-2 (right) with the CubeSat Release Systems (CRS) attached (on top).

During the integration activities first the CRSs were integrated on to the CubeSats as shown in Figure 14. After pertinent checks the CubeSats were loaded into the correspondent deployers as shown in Figure 15. Finally the deployers were installed on the RemoveDebris plat- form performing the necessary mechanical and electrical checks to ensure the correct function of them in space (Figure 16). The ISIPODs were the first payloads to be integrated on the platform. The ISIPODs are in readiness to be actuated in orbit releasing the CubeSats with the required low velocity 
in order to contribute to successfully perform the net and VBN experiments of the RemoveDebris mission.

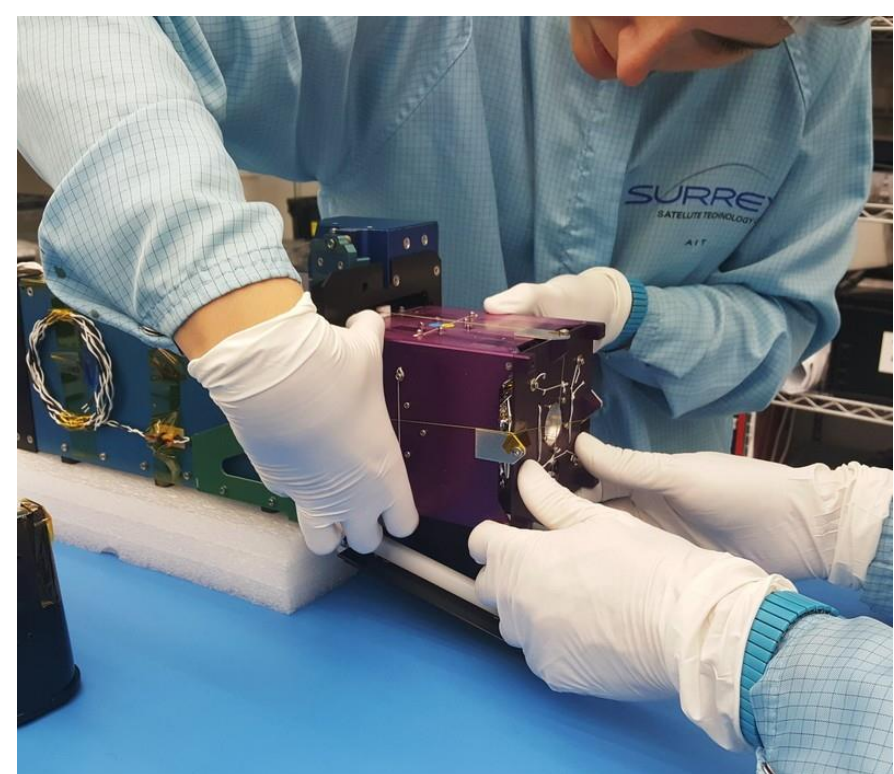

Figure 15: CubeSat insertion into Deployers. This figure shows DS1 being inserted into ISIPOD-1.

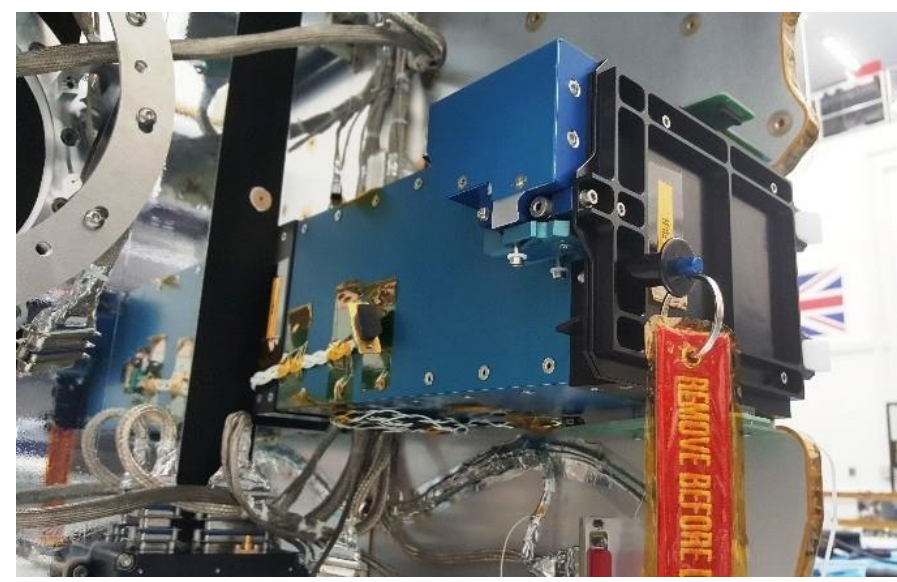

Figure 16: Deployer insertion on to Platform. This figure shows ISIPOD-1 for the net experiment integrated on the platform.

\section{PAYLOAD: NET}

Figure 17 shows the FM net payload. The net consists of a series of ejectable counterweights, that once ejected, draw out the net material. Once the net has captured the CubeSat, a series of times motors in the counterweights reel inwards to draw the net closed. Further design details can be found in [51].

Extensive functional testing has been covered in past research, namely the net deployment on both a Novespace A300 parabolic flight and within the Bremen drop tower; such experiments helped verify requirements R5 and R6. From a functional perspective, once the net hits the target, a series of counterweights with enclosed motors will wrap around the target and will reel in to encapsulate the target. This performance has been demonstrated both in simulation and in functional testing. Further information can be found in $[2,[51]$.

Regarding environmental testing, the acceptance tests comprised functional testing, vibration testing and ther- mal vacuum testing. The functional testing was done just after integration and before and after each environmental test to verify proper functionality. The test simulated the complete mission sequence of the Net Capture Payload in- cluding ejection of lid and net and closure of net via motors. Since progress demonstrated in [52], [6], post-environmental testing, the payload was sent for integration in the platform. The full SEET deployment was conducted using the FM platform but with an EQM net, as the FM is a single-use deployment

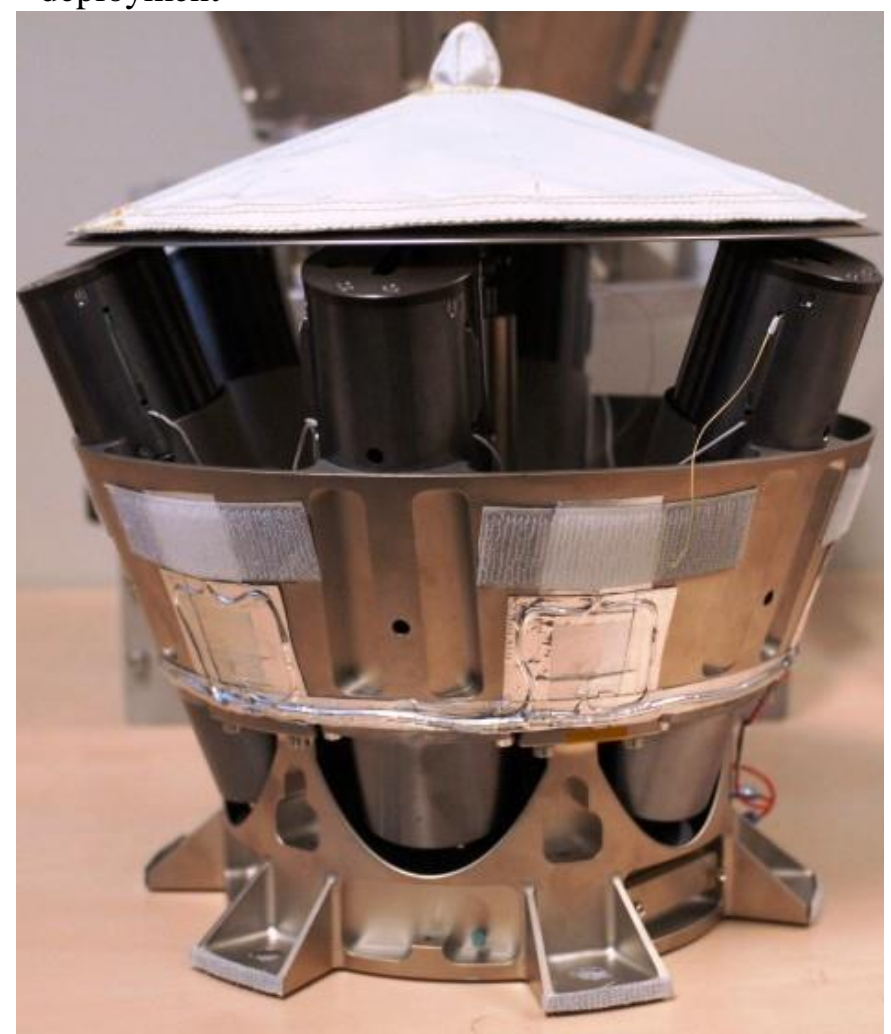

Figure 17: FM Net. This figure shows the final net flight model with ejection springs unloaded.

\section{PAYLOAD: HARPOON TARGET ASSEMBLY (HTA)}

The harpoon target assembly (HTA) can be seen in Figure 18 and the experiment will deploy a target on a carbon fibre boom to $1.5 \mathrm{~m}$ and capture it with a tethered harpoon. The RemoveDebris mission will be the first to test a debris capturing harpoon in space. The system comprises: structure (outer casing), the harpoon (blue, cyan), the cold gas generators which are the ejection canisters for the harpoon (pink), two frangibolts (red) holding the harpoon door and the target panel, the target panel itself (grey), the OSS boom extension unit (green) 
and a kapton safety box (yellow).

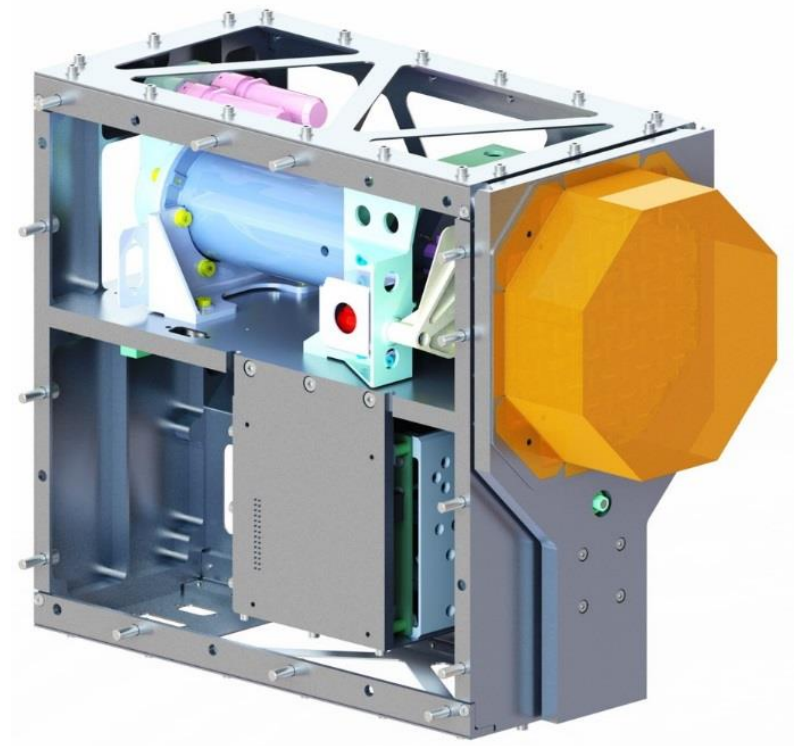

Figure 18: Harpoon: Overall System including Target Assembly Showing: harpoon chamber and mountings (blue, cyan), CGGs (pink), frangibolt (red), OSS boom unit (green), Kapton box (yellow).

When the harpoon hits the target plate, two barbs are deployed; these secure the chaser to the target and allow it to be dragged out of orbit. The experiment will be filmed using high-speed cameras on-board the spacecraft, these will observe the flight of the harpoon and the position of impact.

The completed build can be seen in Figure 19,

Figure 21 and Figure 22.

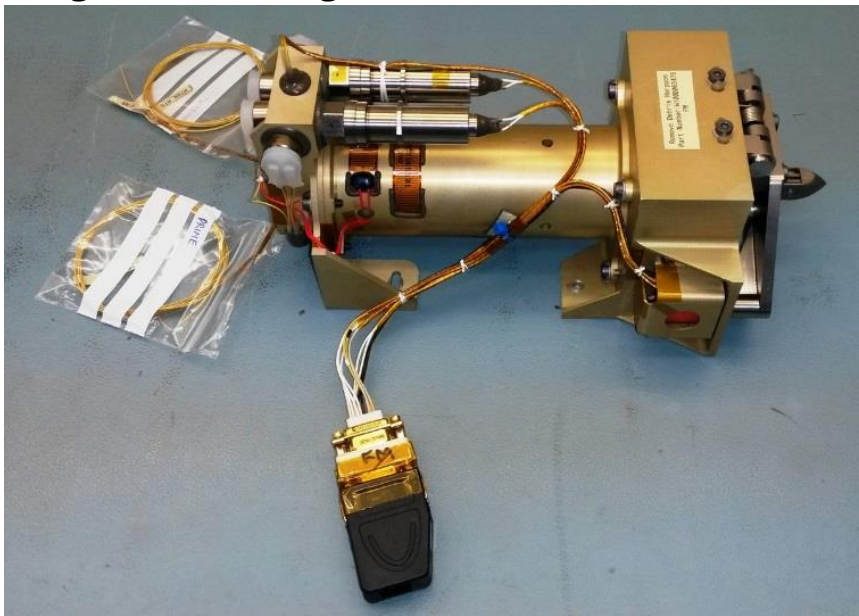

Figure 19: FM Harpoon Payload. Showing the harpoon payload (no MLI) without the harpoon target or casing.

\section{VIII.1. Functional Testing}

The harpoon projectile imparts significant energy into the target material when impacting, even allowing for the energy lost in penetrating the honeycomb panel material. This excess energy (typically up to $20 \mathrm{~J}$ ) may be transmit- ted back through the boom structure. Testing has been conducted to evaluate approaches to absorb this energy, in order to protect the boom. Experiments with flexible couplings between boom and target have been evaluated, as shown in the test in Figure 20.

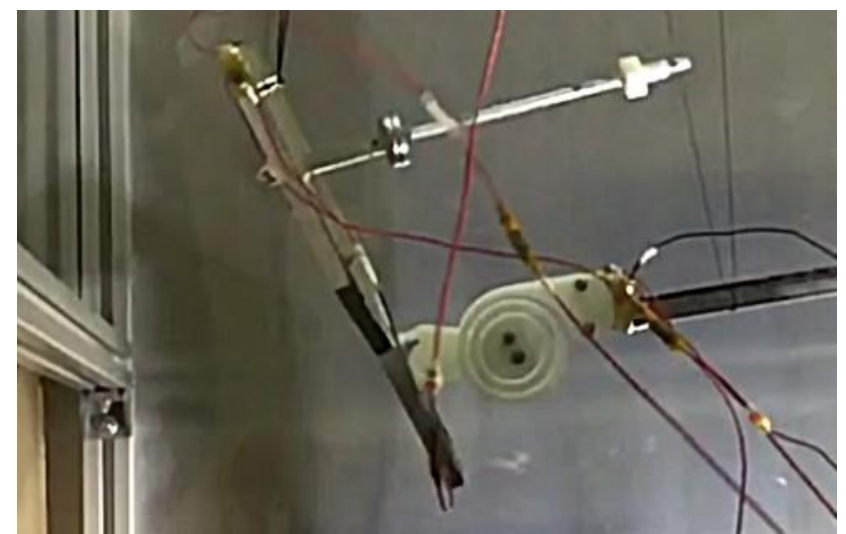

Figure 20: Harpoon: Snail Test. Shows testing with brackets that absorb the shock energy. Showing: harpoon projectile, target frame, end of boom, flexible absorption system and gravity assist lines.

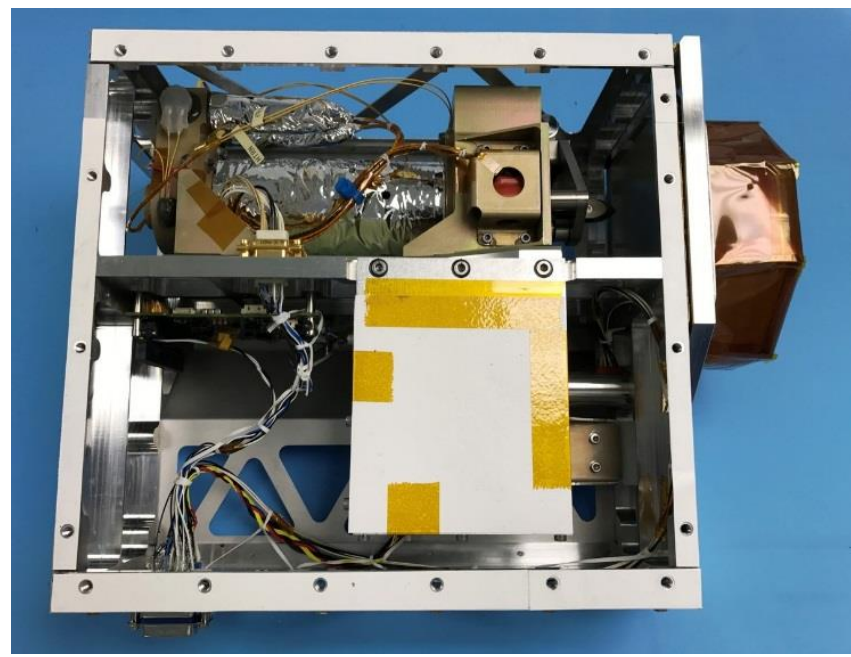

Figure 21: FM HTA - 1. This figure shows the harpoon target assembly (HTA) from the right side.

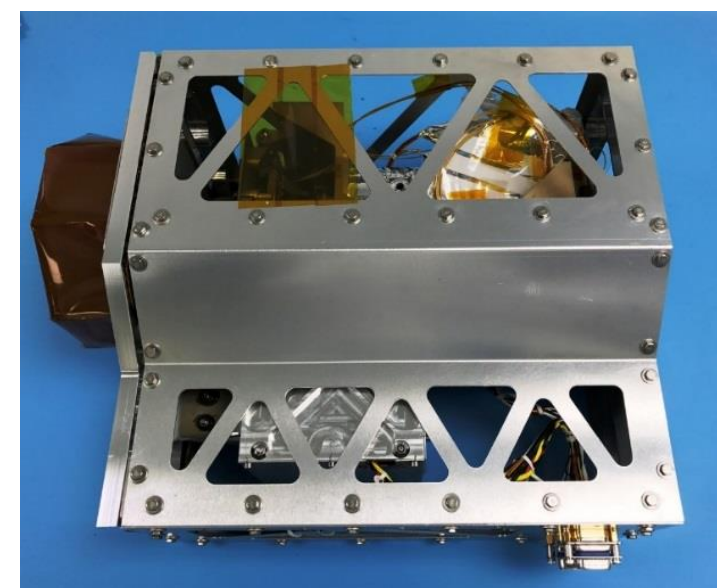

Figure 22: FM HTA - 2. This figure shows the harpoon target assembly (HTA) from the left side. 
VIII.2. Functional Testing - Boom Deployment Tests

Figure 23 shows a boom deployment test, showing the boom in its fully extended position.

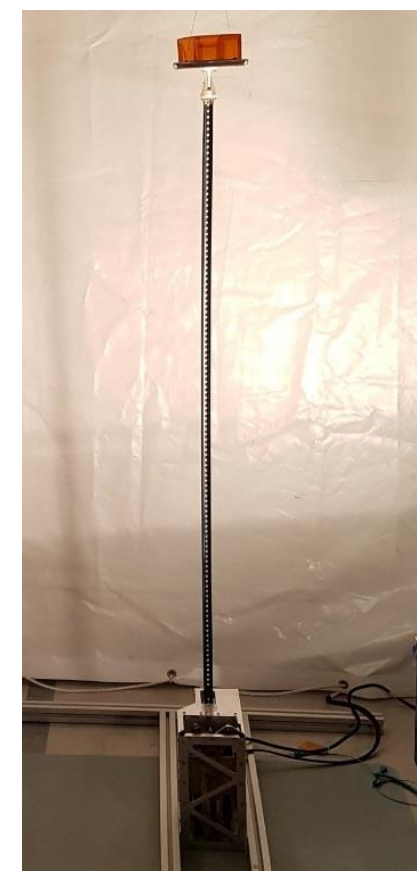

Figure 23: FM Boom - Deployed. This figure shows the harpoon target assembly (HTA) and the boom fully deployed to $1.5 \mathrm{~m}$ with gravity assistance.

\section{VIII.2.1. Functional Testing - Full Firing Tests}

The harpoon is fired by a piston propelled by gas. The point at which the piston releases is determined by a tearpin within the casing, this allows the firing speed to be modified. To verify the harpoon accuracy, multiple firing tests were performed. A laser placed on the tip of the harpoon was used to predict the impact location, once aligned the harpoon was fired upwards into a honeycomb panel. The position of predicted impact was compared to the actual impact in order to characterise the accuracy. An impact can be seen in Figure 24.

The test facility has representative (in-orbit) lighting levels. The ambient light levels have been established (1700 $\mu \mathrm{W} / \mathrm{cm} 2$ visible bandwidth) within the ground test environment to ensure adequate visibility of the projectile $100 \mathrm{~ms}$ flight time with observation cameras. The projectile deployment has been observed to verify correct operation and deployment of the tether slide and tether storage on the spools has been observed to ensure deployment during the early flight phase.

The harpoon strikes the target within a $20 \mathrm{~mm}$ diameter circle, including the size of the harpoon tip. As the target is $100 \mathrm{~mm}$ there is enough margin for variations of the target position in space compared to on ground. A plot of the flight model (FM) impacts can be seen in Figure 25.

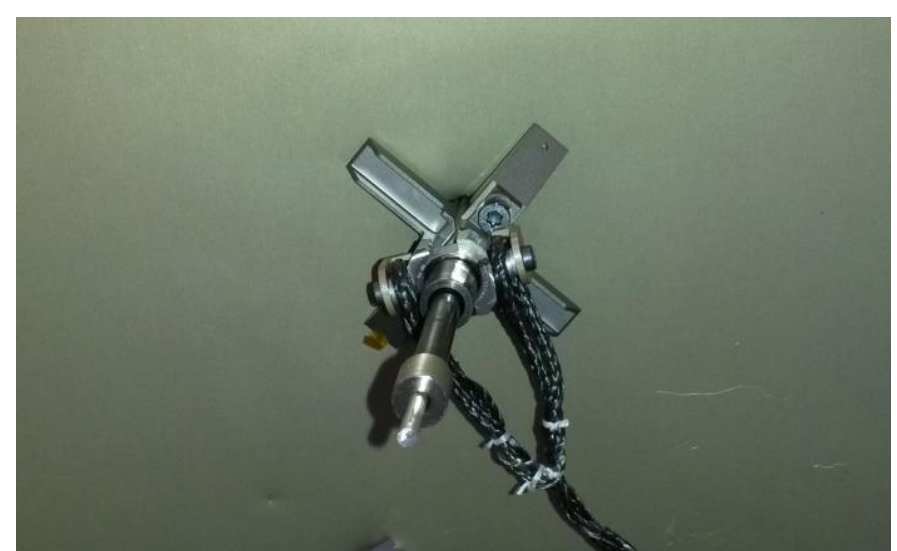

Figure 24: Harpoon Impact. This figure shows a harpoon impacted into a target.

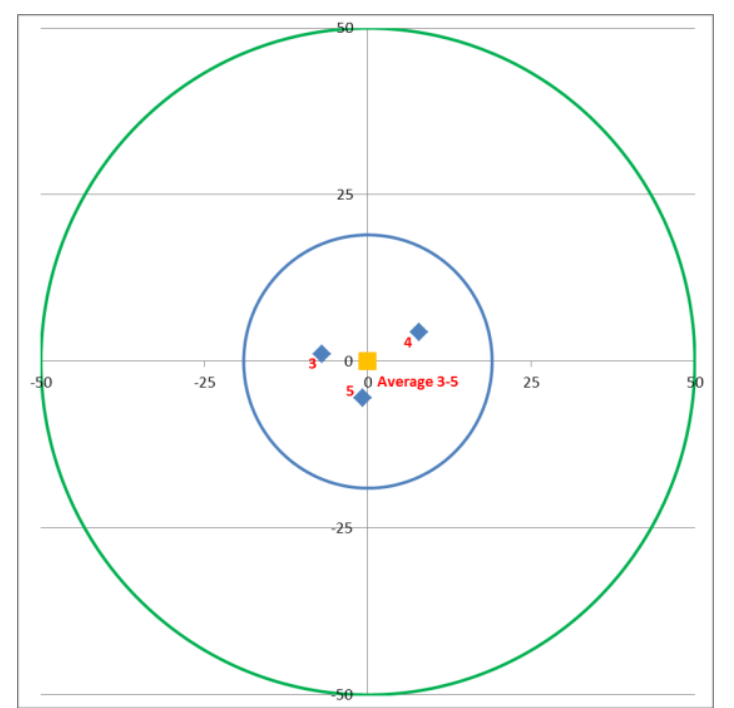

Figure 25: Harpoon Impact Locations. This figure shows the distribution of impact points for the FM. Note that minimal firing tests were performed on the FM compared to the qualification model $(\mathrm{QM})$, preventing excessive wear of the flight firing mechanism.

\section{PAYLOAD: VISION-BASED NAVIGATION (VBN)}

The Vision-Based Navigation is an experiment of proximity navigation between the satellite platform and an artificial mini satellite (DS-2). At the beginning of the experiment DS-2 will be ejected by the platform and will drift gently away for several hours.

The main goal of the experiment is to evaluate navigation algorithms and a VBN sensor. Dedicated image processing and navigation algorithms have indeed been designed at Airbus Defence and Space and INRIA to meet the specific case of non-cooperative rendezvous [25]. Airbus Defence and Space is responsible for the overall VBN experiment and the navigation algorithms, while CSEM is in charge of the sensor.

The sensor has two main subsystems: an off-the-shelf color camera and a flash imaging Light Detection And 
Ranging device (LiDAR) developed by CSEM. Its main functionality is to capture images of DS-2 with both vision- based devices according to a predefined timeline defining snapshot times and integration times. It is foreseen to use the sensor for the harpoon, the net and the VBN experiments. The VBN sensor has the most complex set of functionalities and interface with the S/C amongst the payloads. A proto-flight model (PFM) has been made for the project and can be seen in Figure 28 and Figure 29.

For the latest in VBN developments see [53], [6].

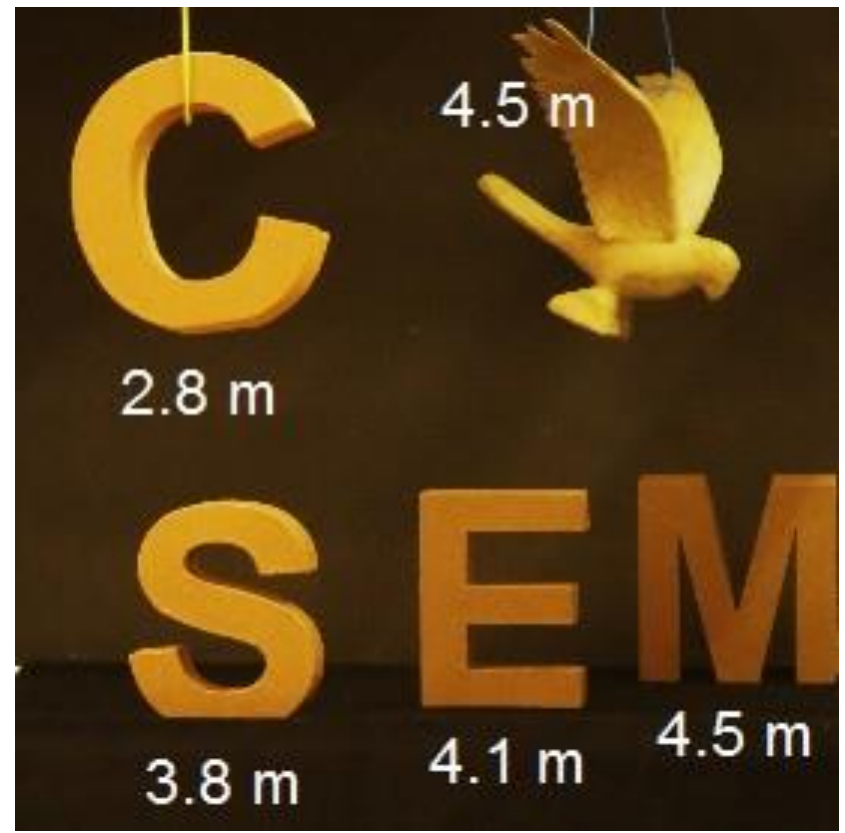

Figure 26: VBN: Image from Camera. Using the letters 'CSEM' from the partner's name. Provides an indication of the targets' distances. From [5]
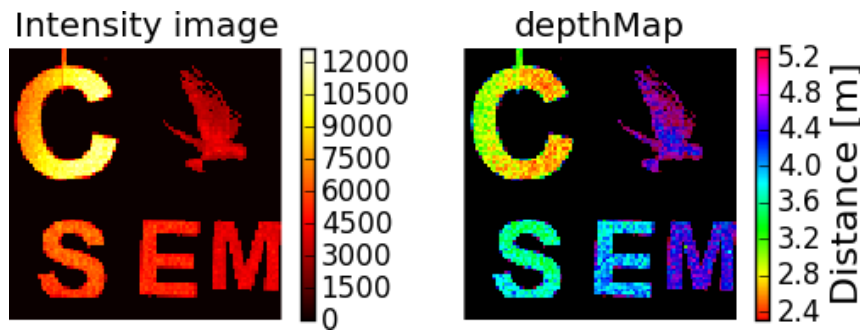

Figure 27: VBN: Image from LiDAR. Left: showing image intensity in number of visible photons (more yellow objects are brighter). Right: 3D depthmap scene in metres. From [5].

\section{IX.0.1. Functional Testing - Imaging}

A set of functional tests have to be conducted with the VBN sensor PFM aiming at taking images with the camera and the LiDAR, and uploading these images from the sensor to an unit simulating the platform PIU.

Figure 26 presents an image captured with the camera. The respective distance of the carton targets are quoted on the image. Figure 27 presents the same scene captured with the LiDAR. The LiDAR provides 2 images: a B\&W intensity image similar to any standard camera, and a distance image or depth map that is a 3D image of the scene of interest or target.

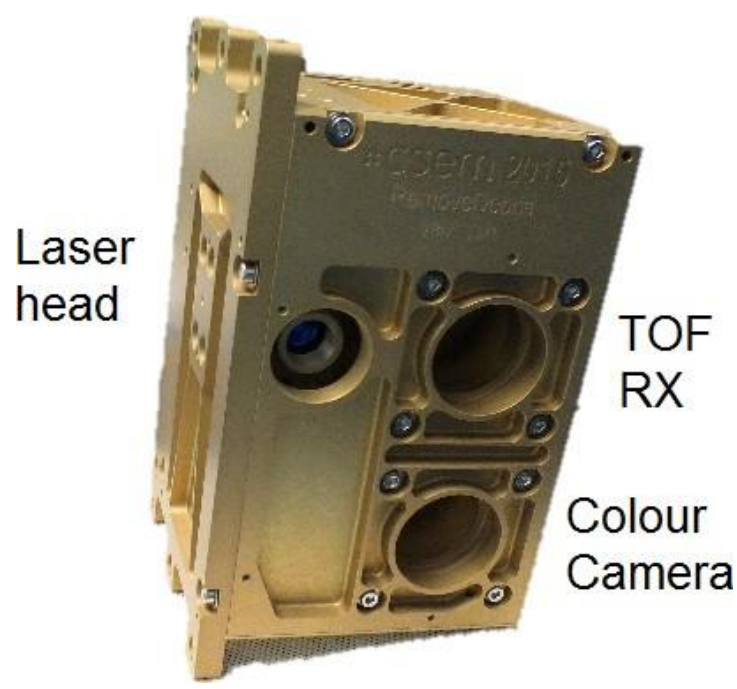

Figure 28: VBN: Sensor PFM.

Following the environmental tests, and before delivery, the PFM is calibrated. The goal is to determine the ge ometrical parameters of both vision-based subsystems to correct optical aberrations.

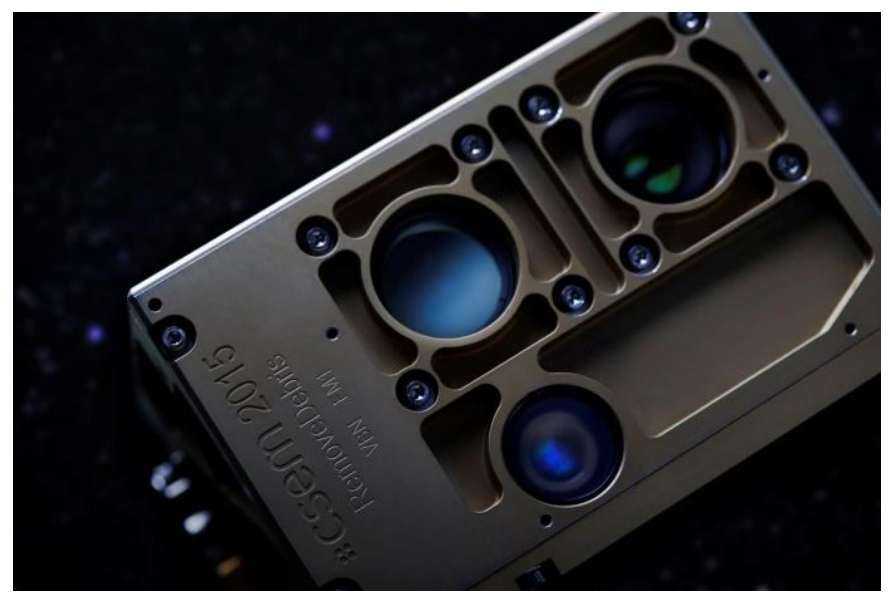

Figure 29: PFM VBN. This figure shows the final VBN flight model.

\section{PAYLOAD: DRAGSAIL}

This section will focus on the testing of the dragsail, both functional and environmental, which is a version of the system used on the InflateSail mission. The dragsail consists of two parts, an inflatable mast and a sail deployment mechanism. The stacked FM, ready for final flight preparation, is visible in Figure 30, where the bottom part is the sail deployment mechanism with deployable carbon fibre booms, the middle is the sail material that is drawn out during sail deployment, and the top part is the inflatable mast that is deployed using a CGG. 


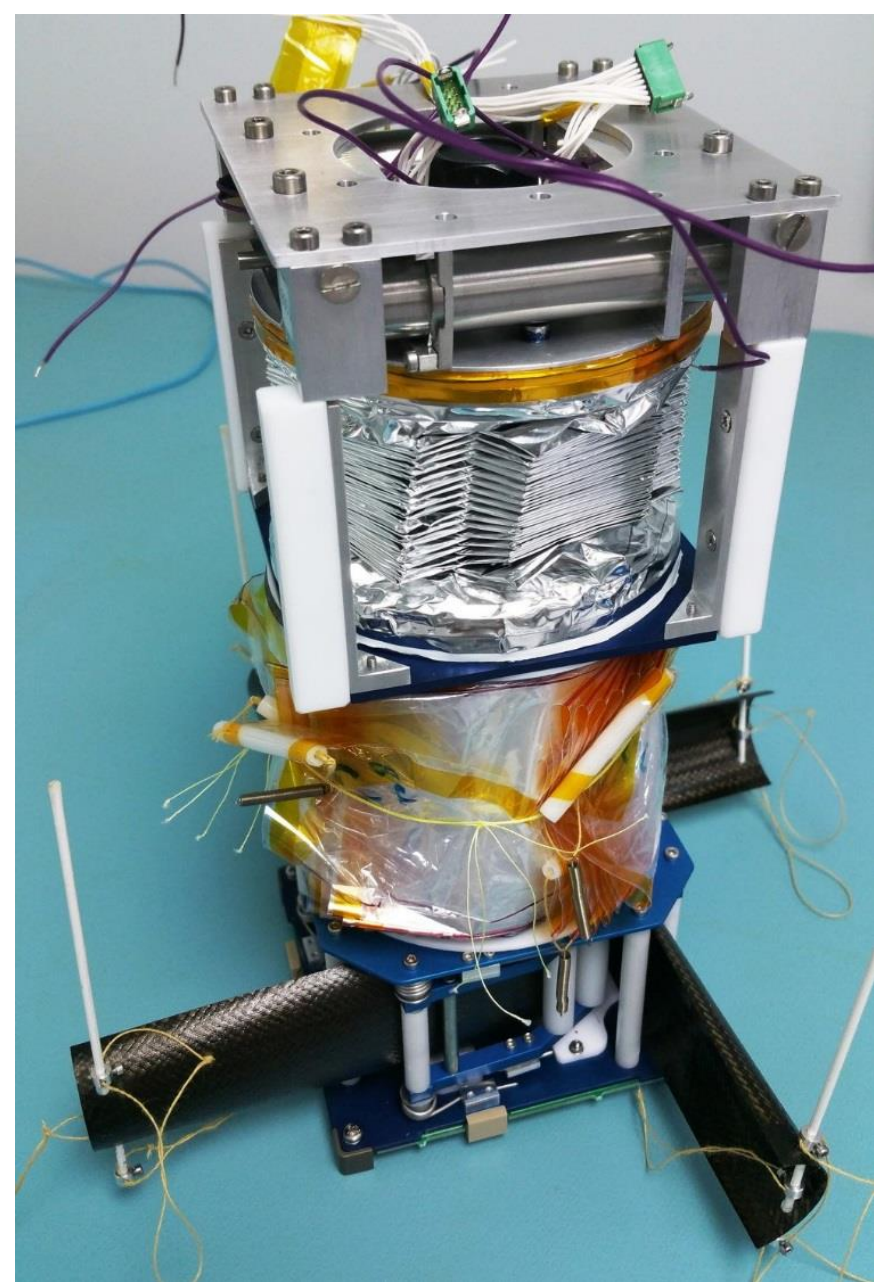

Figure 30: Dragsail: Assembled Flight Payload.

\section{X.1. Functional Testing - Inflation and Sail Deployment}

Several functional tests were performed in inflating the boom and deploying the sail. Initially, inflatable deploy- ment was tested for maximum pressure, under gravity compensation, with a Mylar bladder and finally using a balloon. The balloon test is shown in Figure 31 and shows how the balloon is used as a gravity offloading system.

The removal of the major creases in the skin was clearly observed, showing boom rigidisation.

Figure 32 shows one of the full deployment tests of both mast and sail showing size compliance with requirements R13 and R14.

\section{X.2. Environmental Testing}

A full complement of environmental testing including vacuum, vibration and thermal were performed.

The purpose of the vacuum environmental testing was two-fold. One of the aims was to assess the likelihood of a pressure build-up during ascent to simulate the launch phase and to ensure the solenoid venting valve was correctly operating (the solenoid valve is a normally-open type, so the stowed boom is free to vent until the valve is powered). Secondly, the test helped ensure that the system is airtight in space (a vacuum). During the vacuum testing, a full deployment of the mast was undertaken.
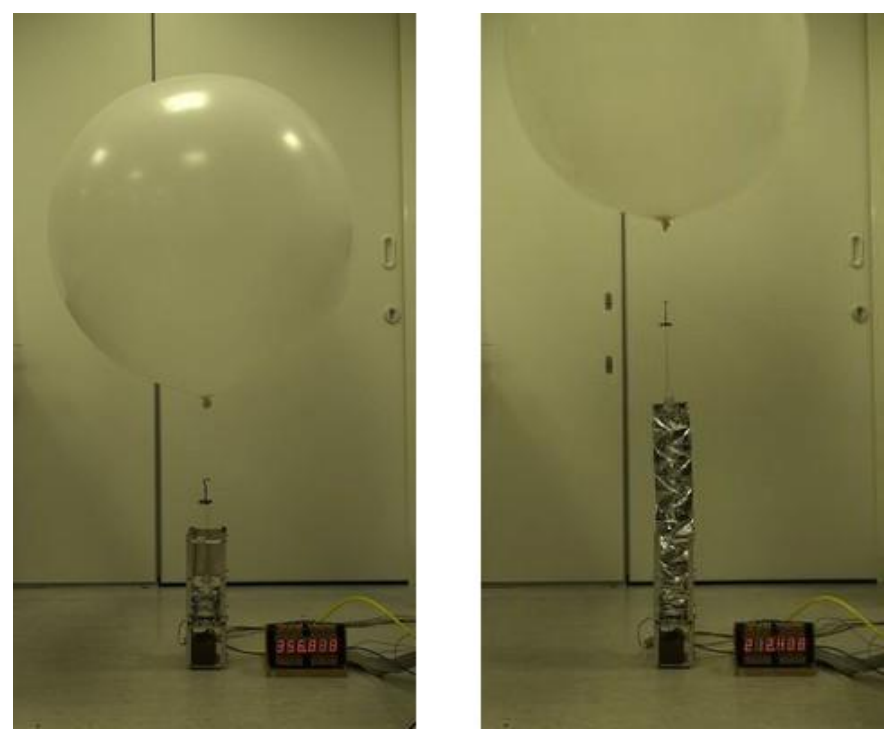

Figure 31: Dragsail: Inflation Test with Balloon Assist. With EQM unit.

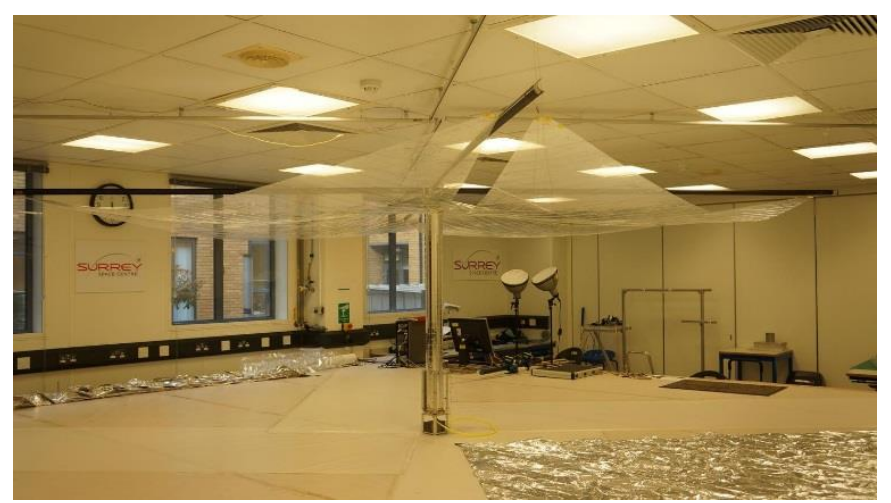

Figure 32: Dragsail: Deployment Test. With EQM unit.

\section{CONCLUSION}

RemoveDebris is aimed at performing key ADR technology demonstrations (i.e. debris observation, capture and de-orbiting) representative of an operational scenario during a low-cost mission using novel key technologies for future missions in what promises to be the first ADR technology mission internationally. The Net experiment has been designed to demonstrate the in-orbit capture of a relatively large debris with a Net, and further developments will be needed to explore how the captured debris can then be retrieved/secured to the mothercraft for its disposal. The Harpoon experiment includes a tether line which could be used for either towing the debris from a mothercraft driven by its propulsion system, or to slow down the debris (passively) using a Dragsail deployed by the mothercraft so that both objects would de-orbit 
together. In this second case the mothercraft would not need a propulsion system. Although the Dragsail is one of the experiments that will be demonstrated by the mission, the issue of the combined dynamics, debrismothercraft during revival is beyond the scope of the current work.

This paper has examined the design of the mission from initial concepts through to manufacture, AIT, testing and up to launch. The complete satellite has now been built, successfully tested, and shipped out to the US in December 2017, for an early 2018 launch to the ISS. From here, through an airlock, the satellite is going to be released in orbit using the ISS robotic arm.

The technologies for debris capture that have been discussed in this article, in the context of ADR represent novel contributions, never attempted before in space. Although there has been a rigorous design, manufacture, assembly, integration and testing, documented in the paper, the ultimate proof of this work will be its deployment and correct functioning in space. This will be the first demonstration of utilising CubeSats as artificial debris targets for capture in space; although this is not a fully-fledged ADR mission as CubeSats are utilised as artificial debris, the project is a very important step towards a fully operational ADR mission.

The mission proposed is a vital prerequisite in achieving the ultimate goal of a cleaner Earth orbital environment

\section{ACKNOWLEDGEMENT} $\mathrm{S}$

This research is supported by the European Commission FP7-SPACE-2013-1 (project 607099) 'RemoveDebris - A Low Cost Active Debris Removal Demonstration Mission', a consortium partnership project consisting of: Surrey Space Centre (University of Surrey), SSTL, Airbus GmbH, Airbus SAS, Airbus Ltd, Ariane Group, Innovative Solutions in Space (ISIS), CSEM, Inria, Stellenbosch University.

\section{REFERENCES}

[1] G.S. Aglietti et al. "The active space debris removal mission RemoveDebris. Part 2: in orbit operations", submitted Acta Astronautica.

[2] Main Website, www.surrey.ac.uk/ssc/research/space vehicle_control/removedebris/.

[3] J. L. Forshaw, G. S. Aglietti, N. Navarathinam, H. Kadhem, T. Salmon, A. Pisseloup, E. Joffre, T. Chabot, I. Retat, R. Axthelm, S. Barraclough, A. Ratcliffe, C. Bernal, F. Chaumette, A. Pollini, W. H. Steyn, RemoveDEBRIS: An in-orbit active debris removal demonstration mission, Acta Astronautica 127 (2016) 448 - 463. doi:10.1016/j.actaastro.2016.06.018.

[4] E. Joffre, J. L. Forshaw, T. Secretin, S. Reynaud, T. Salmon, A. Pisseloup, G. Aglietti, RemoveDebris - mission analysis for a low cost active debris removal demonstration in 2016, in: 25th International Symposium on Space Flight Dynamics (ISSFD), Munich, Germany, 2015.

[5] J. L. Forshaw, G. S. Aglietti, T. Salmon, I. Retat, M. Roe, T. Chabot, C. Burgess, A. Pisseloup, A. Phipps, C. Bernal, F. Chaumette, A. Pollini, W. H. Steyn, Review of final payload test results for the RemoveDebris active debris removal mission, in: 67th International Astronautical Congress, Guadalajara, Mexico, 2016.

[6] J. L. Forshaw, G. Aglietti, T. Salmon, I. Retat, M. Roe, C. Burgess, T. Chabot, A. Pisseloup, A. Phipps, C. Bernal, F. Chaumette, A. Pollini, W. H. Steyn, Final payload test results for the RemoveDebris active debris removal mission, Acta Astronautica 138 (2017) 326 - 342. doi:10.1016/j.actaastro. 2017.06.003.

[7] J. L. Forshaw, G. Aglietti, T. Salmon, I. Retat, C. Burgess, T. Chabot, A. Pisseloup, A. Phipps, C. Bernal, F. Chaumette, A. Pollini, W. H. Steyn, The RemoveDebris ADR mission: preparing for an international space station launch, in: ESA 7th European Conference on Space Debris, ESOC, Germany, 2017.

[8] R. Biesbroek, A. Wolahan, Maturing the technology for ESA's e.Deorbit mission to remove a large, heavy space debris from low earth orbit, in: CNES 4th International Workshop on Space Debris Modelling and Remediation, Paris, France, 2016.

[9] L. Innocenti, Clean space - an overview, in: ESA Clean Space Industrial Days, ESTEC, Netherlands, 2016.

[10] C. Saunders, J. L. Forshaw, V. J. Lappas, A. Chiesa, B. Parreira, R. Biesbroek, Mission and systems design for the debris re- moval of massive satellites, in: 65th International Astronautical Congress, Toronto, Canada, 2014.

[11] C. Saunders, J. L. Forshaw, V. J. Lappas, D. Wade, D. Iron, R. Biesbroek, Business and economic considerations for service oriented active debris removal missions, in: 65th International Astronautical Congress, Toronto, Canada, 2014

[12] S. Estable, Envisat removal by robotic capture means - results of the airbus ds led e.Deorbit Phase B1 ESA study, in: ESA Clean Space Industrial Days, ESTEC, Netherlands, 2016.

[13] M. Scheper, e.deorbit Phase B1 âĂŞ System Overview (OHB), in: ESA Clean Space Industrial Days, ESTEC, Netherlands, 2016.

[14] C. Cougnet, B. Gerber, C. Heemskerk, K. Kapellos, G. Visentin, On-orbit servicing system of a GEO satellite fleet, in: 9th ESA Workshop on Advanced Space Technologies for Robotics and Automation 'ASTRA 2006', ESTEC, Netherlands, 2006.

[15] A. Pisseloup, T. Salmon, C. Cougnet, M. Richard, ADR con- cepts from CNES funded study OTV, in: 64th International Astronautical Congress, Beijing, China, 2013.

[16] D. Reintsema, B. Sommer, T. Wolf, J. Theater, A. Radthke, W. Naumann, P. Rank, J. Sommer, DEOS - the in-flight technology demonstration of german's robotics approach to dis- pose malfunctioned satellites, in: ESA 11th Symposium on Ad- vanced Space Technologies in Robotics and Automation, ESTEC, Netherlands, 2011.

[17] Astrium Space Transportation, ROGER Phase-A Final Report Executive Summary, Tech. Rep. ROG-SIBRE-EXS, Astrium Space Transportation (2003).

[18] A. Pisseloup, S. Estable, K. Pegg, E. Ferreira, R. Delage, J.-M Pairot, T. Salmon, A. Ratcliffe, M. Frezet, Airbus defence and space's vision and activities in active debris removal and on-orbit servicing, in: CNES 4th International Workshop on Space Debris Modelling and Remediation, Paris, France, 2016.

[19] M. Merino, E. Ahedo, C. Bombardelli, H. Urrutxua, J. Pelaez, L. Summerer, Space debris removal with an ion beam shep- herd satellite: target-plasma interaction, in: 47th AIAA Joint Propulsion Conference \& Exhibit, San Diego, US, 2011.

[20] A. Parness, Orbital debris removal with gecko-like adhesives; technology development and mission design, in: 66th International Astronautical Congress, Jerusalem, Israel, 2015.

[21] C.Trentlage, E.Stoll, The applicability of gecko adhesives in a docking mechanism for active debris removal missions, in: 13th Symposium on Advanced Space Technologies in Robotics and Automation, ASTRA 2015, ESTEC, Netherlands, 2015.

[22] A. Chiesa, G. Gambacciani, D. Renzoni, G. Bombaci, Enabling technologies for active space debris removal: the CADET (CAp- 
ture and DEorbiting Technologies) project, in: CNES 4th International Workshop on Space Debris Modelling and Remediation, Paris, France, 2016.

[23] M. Bicocca, Debris capture technologies overview, Tech. rep., Aviospace (May 2014).

[24] A. Petit, E. Marchand, K. Kanani., Tracking complex targets for space rendezvous and debris removal applications, in: IEEE/RSJ Conference on Intelligent Robots and Systems, IROS'12, Vilamoura, Portugal, 2012.

[25] T. Chabot, E. Kervendal, N. Despre, K. Kanani, P. Vidal, E. Monchieri, D. Rebuffat, S. Santandrea, J. L. Forshaw, Relative navigation challenges and solutions for autonomous orbital rendezvous, in: EuroGNC 2015, Toulouse, France, 2015.

[26] A. Yol, E. Marchand, F. Chaumette, K. Kanani, T. Chabot, Vision-based navigation in low earth orbit, in: i-SAIRAS 2016, Beijing, China, 2016.

[27] A. Sonnenburg, Image recognition and processing for navigation (irpn), in: ESA Clean Space Industrial Days, ESTEC, Nether- lands, 2016.

[28] N. Deslaef, J. Christy, Rendezvous sensors and navigation, in: ESA Clean Space Industrial Days, ESTEC, Netherlands, 2016.

[29] Jena-Optronik, The rvs3000 and rvs3000-3d lidar sensors for rendezvous and docking and space robotics, in: ESA Clean Space Industrial Days, ESTEC, Netherlands, 2016.

[30] J. L. Forshaw, C. Massimiani, M. Richter, A. Viquerat, E. Simons, R. Duke, G. Aglietti, Surrey Space Centre: A survey of debris removal research activities, in: 66th International Astronautical Congress, Jerusalem, Israel, 2015.

[31] Underwood, C, Denis, A, Viquerat, AD, Taylor, B, Sanders, B, Stewart, B, Massimiani, C, Bridges, C, Masutti, D, Aglietti, G, Schenk, M, Duke, R \& Fellowes, S, 2019, 'InflateSail De-Orbit Flight Demonstration Results and Follow-On Drag-Sail Applications'. Acta Astronautica.

[32] P. Voigt, C. Vogt, B. Barthen, H. Stokes, C. Underwood, A. Knoll, K. Ryden, M. Macdonald, E. Kerr, et. al., TeSeR - technology for self-removal - a horizon 2020 project to ensure the post-mission-disposal of any future spacecraft, in: ESA Clean Space Industrial Days, ESTEC, Netherlands, 2016.

[33] S. Antonetti, D-SAT mission: An in-orbit demonstration of autonomous decommissioning capabilities in changing space debris mitigation requirements scenario, in: CNES 4th International Workshop on Space Debris Modelling and Remediation, Paris, France, 2016.

[34] I. A. Sanchez, D. Paris, F. Allard, N. Frischauf, The navigation and communication systems for the Automated Transfer Vehicle, in: IEEE 49th Vehicular Technology Conference, Vol. 2, 1999, pp. 1187-1192. doi:10.1109/VETEC.1999.780535.

[35] K. Yoshida, ETS-VII Flight Experiments For Space Robot Dynamics and Control, Vol. 271, Experimental Robotics VII, Springer, 2001.

[36] S. Clark, Japanese Cargo Ship Ends Mission after Space Debris Experiment Flounders, spaceflightnow.com, 2017.

[37] M. Richard, L. Kronig, F. Belloni, S. Rossi, V. Gass, C. Paccolat, J. Thiran, S. Araomi, I. Gavrilovich, H. Shea, Uncooperative rendezvous and docking for microsats: The case for CleanSpace One, in: 6th International Conference on Recent Advances in Space Technologies (RAST), Istanbul, Turkey, 2013.

[38] B. Gorret, L. MÃl'trailler, L. Moreau-Gentien, P.-A. MÃd'usli, A. Guignard, M. Richard, M. Lauria, Status of the development of the CleanSpace One capture system, in: CNES 4th Interna- tional Workshop on Space Debris Modelling and Remediation, Paris, France, 2016.

[39] N. Okada, ADRAS 1: Spacecrafts EOL solutions and debris removal, in: CNES 4th International Workshop on Space Debris Modelling and Remediation, Paris, France, 2016.

[40] M. Okada, A. Okamoto, K. Fujimoto, M. Ito, Maximizing post mission disposal of mega constellations satellites reaching end of operational lifetime, in: ESA 7th European Conference on Space Debris, ESOC, Germany, 2017.
[41] European Space Agency, e.Deorbit CDF Study Report, Tech. Rep. CDF-134(A), European Space Agency (2012).

[42] NanoRacks, Space station CubeSat deployment services, Tech. rep. (February 2015)

[43] Le Fevre et al., Compliance of disposal orbits with the French Space Act: the good practices and the STELA tool, Acta Astro- nautica 94 (1).

[44] Fraysse et al., Long term orbit propagation techniques developed in the frame of the French Space Act, Journal of Aerospace Engineering, Sciences and Applications 4 (4).

[45] C. Bonnal, CNES activities related to space debris, in: $\mathrm{P}^{\mathbf{2}}$ ROTECT workshop, Torino, Italy, 2013.

[46] C. Massimiani, J. L. Forshaw, G. S. Aglietti, Cubesats as artifi- cial debris targets for active debris removal missions, in: Stardust Final Conference, ESTEC, Netherlands, 2016.

[47] J. L. Forshaw, The RemoveDebris ADR mission: Overview of cubesat 'artificial debris' targets, in: CNES 4th International Workshop on Space Debris Modelling and Remediation, Paris, France, 2016.

[48] L. Visagie, J. L. Forshaw, T. E. Frame, V. J. Lappas, W. H. Steyn, A miniaturised attitude control and determination sys- tem for the QB50 and SME-SAT missions, in: ESA Guidance, Navigation, and Control Conference, Porto, Portugal, 2014.

[49] R. Duke, C. P. Bridges, B. Steward, B. Taylor, C. Massimiani, J. Forshaw, G. Aglietti, Integrated flight and ground software framework for fast mission timelines, in: 67th International Astronautical Congress, Guadalajara, Mexico, 2016.

[50] G. Secheli, A. D. Viquerat, G. S. Aglietti, Mechanical devel-opment of a novel inflatable and rigidizable structure, in: 3rd AIAA Spacecraft Structures Conference, San Diego, US, 2016.

[51] R. Axthelm, B. Klotz, I. Retat, U. Schlossstein, W. Tritsch, S. Vahsen, Net capture system for debris removal demonstration mission, in: 7th European Conference on Space Debris, ESOC, Darmstadt, Germany, 2017.

[52] R. Axthelm, B. Klotz, I. Retat, U. Schlossstein, W. Tritsch, S. Vahsen, Net capture mechanism for debris removal demonstration mission, in: ESA 7th European Conference on Space Debris, ESOC, Germany, 2017.

[53] T. Chabot, K. Kanani, A. Pollini, F. Chaumette, E. Marchand, J. Forshaw, Vision-based navigation experiment on-board the RemoveDebris mission, in: GNC 2017: 10th International ESA Conference on GNC, Salzburg, Austria, 2017. 